INEEL/EXT-01-00173

February 2001

\title{
Process Options Description for Vitrification Flowsheet Model of INEEL Sodium Bearing Waste
}
T. T. Nichols
D. D. Taylor
L. Lauerhass
C. M. Barnes 


\section{DISCLAIMER}

This information was prepared as an account of work sponsored by an agency of the U.S. Government. Neither the U.S. Government nor any agency thereof, nor any of their employees, makes any warranty, express or implied, or assumes any legal liability or responsibility for the accuracy, completeness, or usefulness of any information, apparatus, product, or process disclosed, or represents that its use would not infringe privately owned rights. References herein to any specific commercial product, process, or service by trade name, trademark, manufacturer, or otherwise, does not necessarily constitute or imply its endorsement, recommendation, or favoring by the U.S. Government or any agency thereof. The views and opinions of authors expressed herein do not necessarily state or reflect those of the U.S. Government or any agency thereof. 
INEEL/EXT-01-00173

Revision 0

\title{
Process Options Description for Vitrification Flowsheet Model of INEEL Sodium Bearing Waste
}

\author{
Todd T. Nichols \\ Dean D. Taylor \\ Lance Lauerhass \\ Charles M. Barnes
}

Published February 2001

Idaho National Engineering and Environmental Laboratory High Level Waste Program

Idaho Falls, Idaho 83415

Prepared for the

U.S. Department of Energy

Assistant Secretary for

Environmental Management

Under DOE Idaho Operations Office

Contract DE-AC07-99ID13727 


\section{TABLE OF CONTENTS}

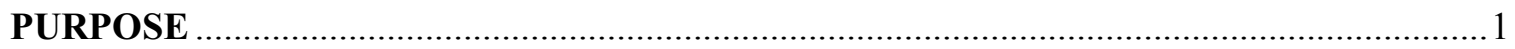

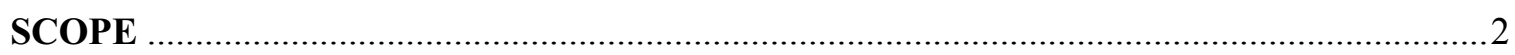

OBJECTIVES

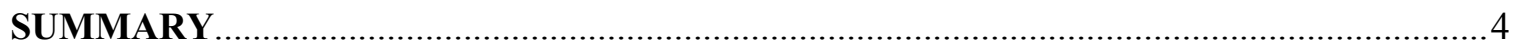

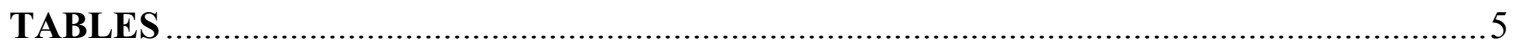

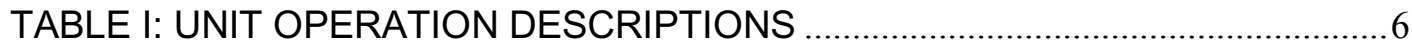

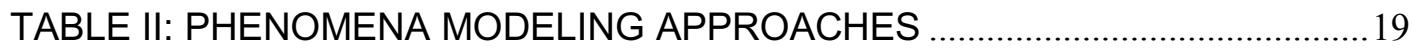

TABLE III: REPRESENTATIVE COMPOSITIONS OF DILUTE SBW FEED ...........36

TABLE IV: REPRESENTATIVE COMPOSITIONS IN SBW TANK..........................40

TABLE V: REPRESENTATIVE GLASS COMPOSITION ........................................44

TABLE VI: MISCELLANEOUS MASS BALANCE ASSUMPTIONS ........................43

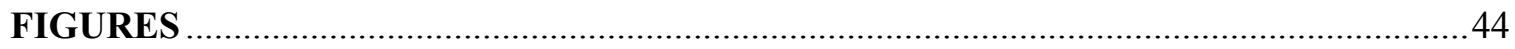

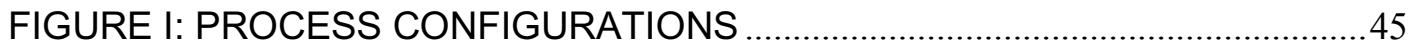

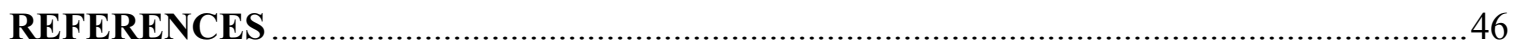




\section{PURPOSE}

The purpose of this document is to provide the technical information to Savannah River Site (SRS) personnel that is required for the development of a basic steady-state process simulation of the vitrification treatment train of sodium bearing waste (SBW) at Idaho National Engineering and Environmental Laboratory (INEEL). INEEL considers simulation to have an important role in the integration/optimization of treatment process trains for the High Level Waste (HLW) Program. This project involves a joint Technical Task Plan (TTP ID77WT31, Subtask C) between SRS and INEEL. The work scope of simulation is different at the two sites. This document addresses only the treatment of SBW at INEEL. The simulation model(s) is to be built by SRS for INEEL in FY-2001. 


\section{SCOPE}

This document provides all the information required a priori for SRS engineers to construct and link unit operation modules in a commercial software simulation application to represent the treatment train for the vitrification of SBW. The information required is of a mid- to high-level nature and consists of the following: 1) a list of specific unit operations, their operating conditions and constraints, and the modeling approaches that will be used in FY-2001 (see Tables I and II); 2) several potential configurations of the unit operations, and their interdependencies via stream connections (see Figure I); and 3) stream compositional makeups (see Table III $\mathrm{VI})$.

Low-level information, such as assumed partition fractions or separation efficiencies, is not detailed in this document because it is not required for the construction and verification of the simulation. The users' manual supplied by SRS will list values for and explain all such low-level assumptions involved with the simulation's data packs created by SRS. INEEL personnel will coordinate with SRS when selecting values to use for the low-level data during the verification phase of the simulation. The document Savannah River Technology Center (2001) discusses the quality assurance plan for the simulation model.

This document is focused only on the steady-state simulation of the direct vitrification of SBW to be performed in FY-2001. The treatment of newly generated liquid waste (NGLW), tank heels, and solid calcine will not be modeled with the process simulation package in FY-2001, and thus, they are outside the scope of this document. 


\section{OBJECTIVES}

The purpose of building the simulation model in a commercial software package in FY-2001 is to add thermodynamic equilibrium to the simulation capabilities of the INEEL HLW Program. The modeling of INEEL SBW vitrification is currently performed with linked EXCEL spreadsheets and lacks a physical properties database. The objective for FY-2001 is to perform basic mass and heat balances on individual unit operations and around the entire treatment train. Having an integrated model with a thermodynamic database will increase the ability of INEEL engineers to assess the system-wide impacts of local changes at individual unit operations.

Such capability will help to reduce the life-cycle schedules and costs of HLW projects, which is imperative given the Settlement Agreement commitments and shrinking DOE budgets. There is currently no funding for an integrated pilot plant demonstration of the treatment train, which increases the role of process simulation. Refinements to the physical properties database and unit operation models are planned for the out-years to support future optimization efforts and detailed design of SBW treatment.

Simulation results will be used by engineers and scientists to develop higher-level recommendations for DOEID and INEEL program managers regarding the treatment of HLW. As such, it is expected that technical personnel will be the primary end-users of the simulation results, and program management will be an indirect end-user. Simulation will not be used as a formal part of waste form qualification (Nichols, 2000). The reader can refer to Barnes, Lauerhass, Nichols, and Taylor (2000) to learn how the simulation efforts of this project being performed in FY-2001 relate to the project's entire scope and objectives. 


\section{SUMMARY}

Three potential process configurations will be modeled in the simulator in FY-2001. These are shown in Figure I. All differences between the three configurations reside in the offgas treatment section of the process train. The three configurations were selected to form a reasonable set of unit operations to help assure that INEEL will have models for the process configuration eventually chosen for direct vitrification of SBW. SRS engineers will build three models in the commercial simulation package, one for each configuration.

Table I describes the details of the individual unit operations. A brief overview of each operation is provided, along with the natural phenomena involved, how the phenomena are currently modeled in INEEL's spreadsheet model, the modeling approach SRS plans to use in FY-2001, and constraints/targets. Table II describes the modeling approaches possible for each of the phenomena mentioned in Table I.

Representative compositional makeups of several streams are given in Tables III - VI. The amount of stream information is provided not to over-constrain the simulation results but rather to facilitate a step-wise construction of the unit operations and debugging. 


\section{TABLES}

Table I: Unit Operation Descriptions

Table II: Phenomena Modeling Approaches

Table III: Representative Compositions of Dilute SBW Feed

Table IV: Representative Compositions in SBW Tank

Table V: Representative Gas Streams

Table VI: Miscellaneous Mass Balance Assumptions 


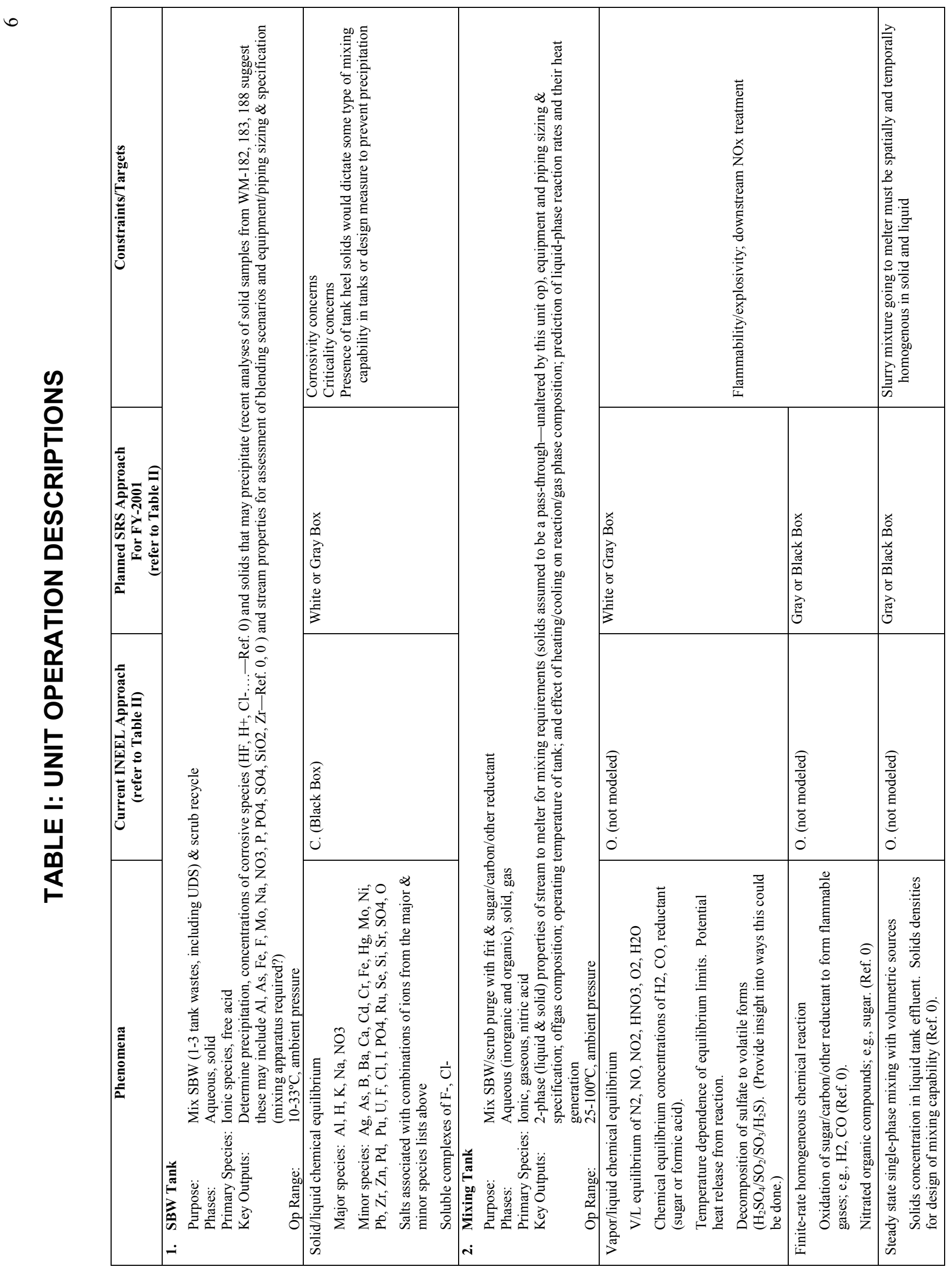




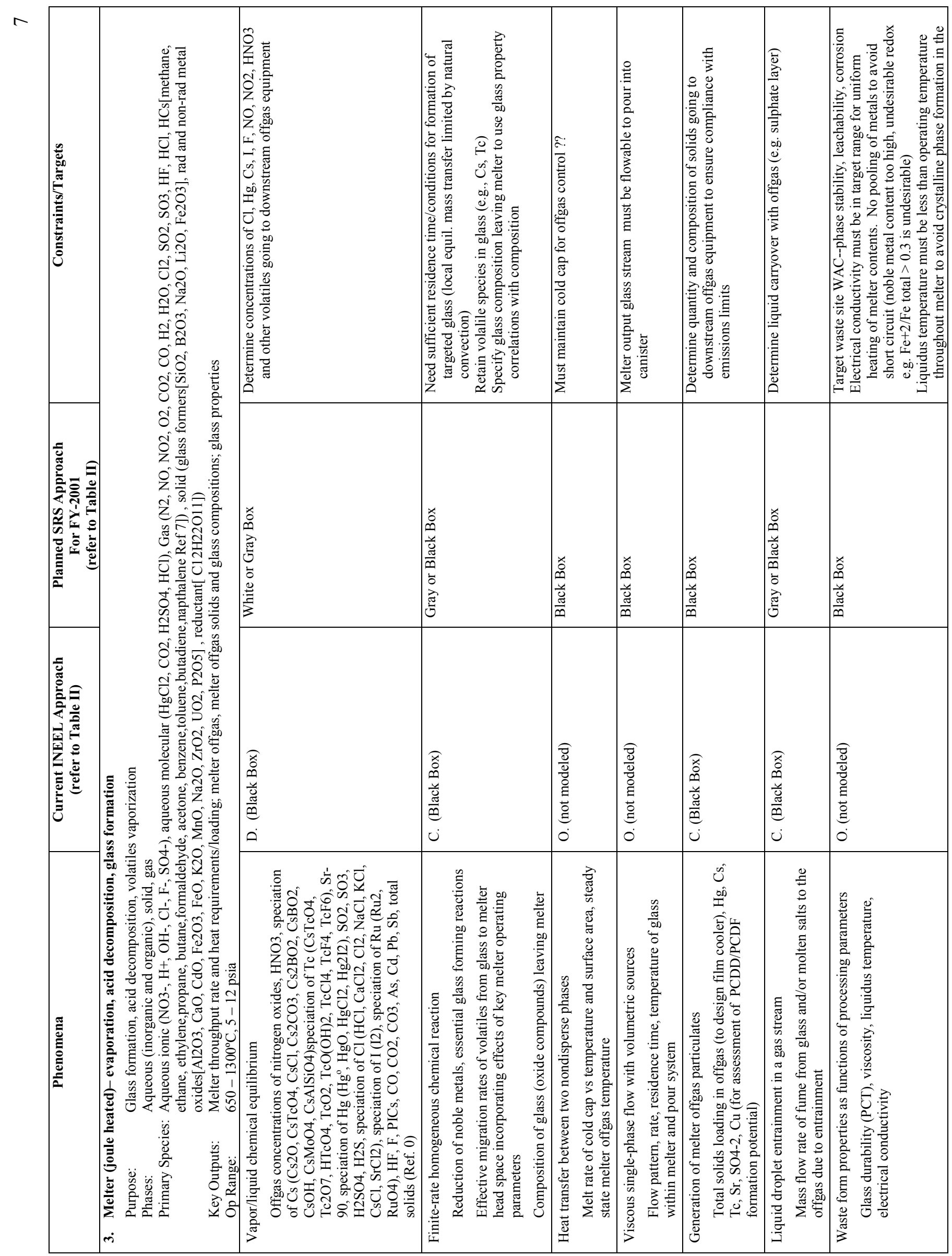




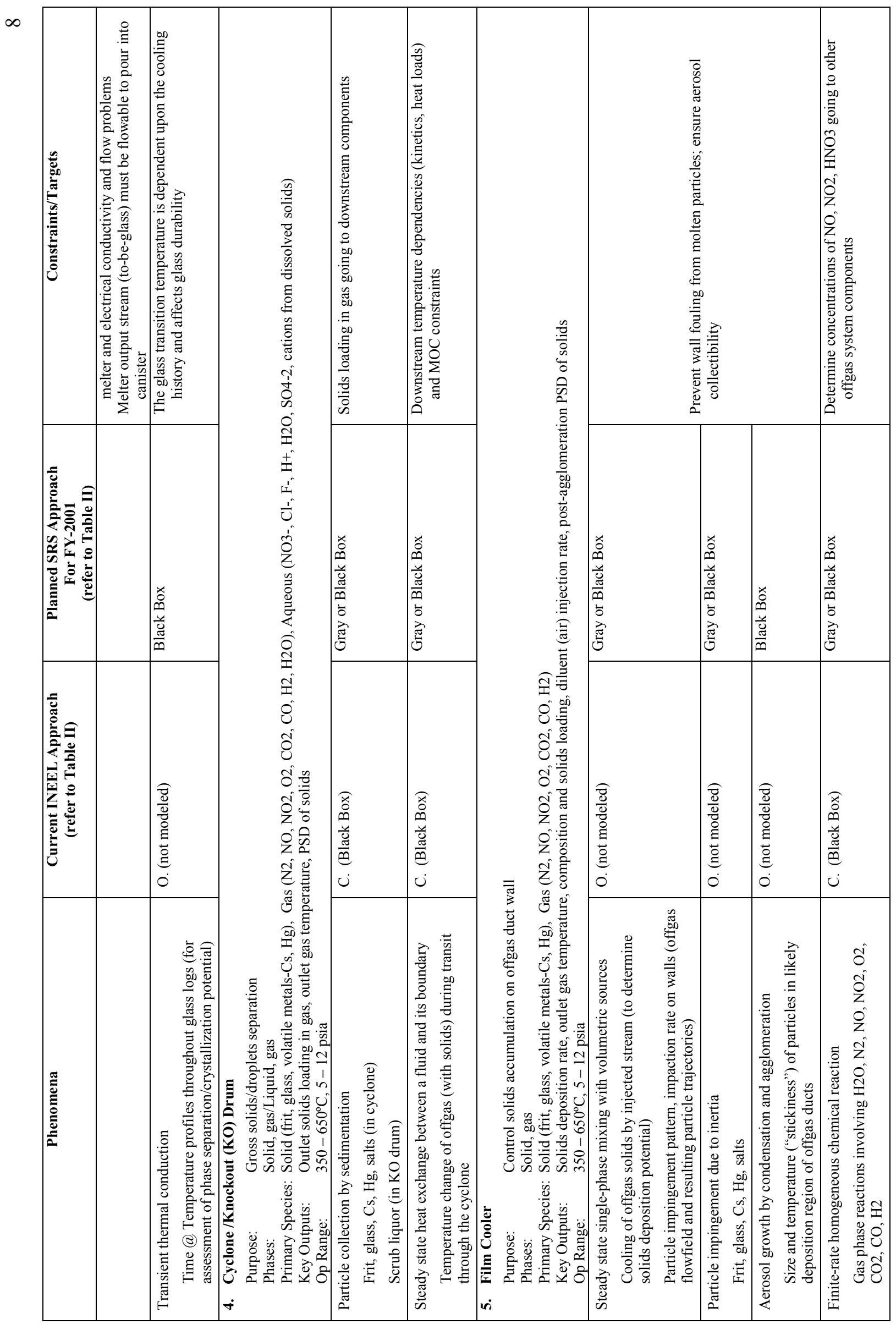




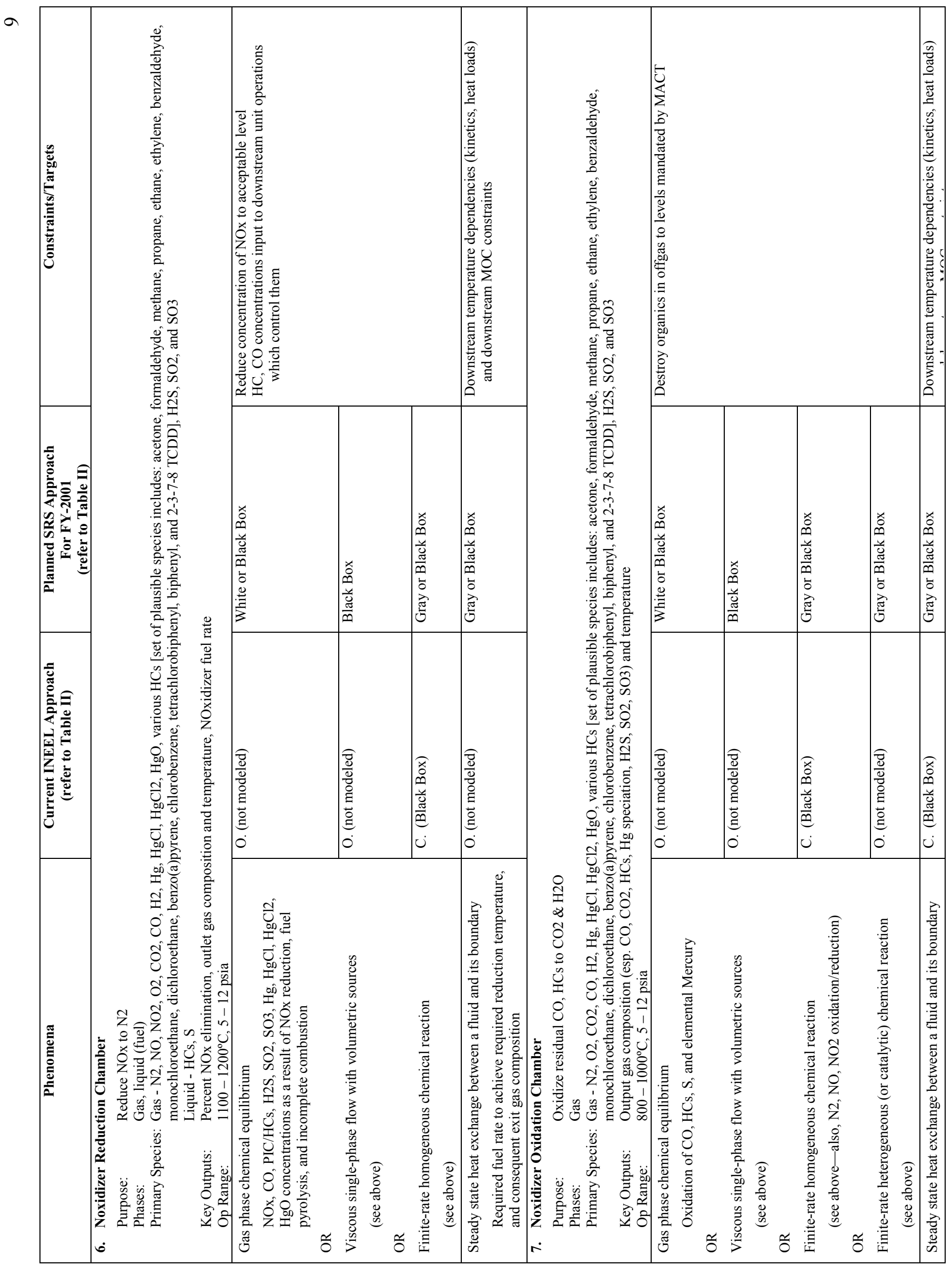




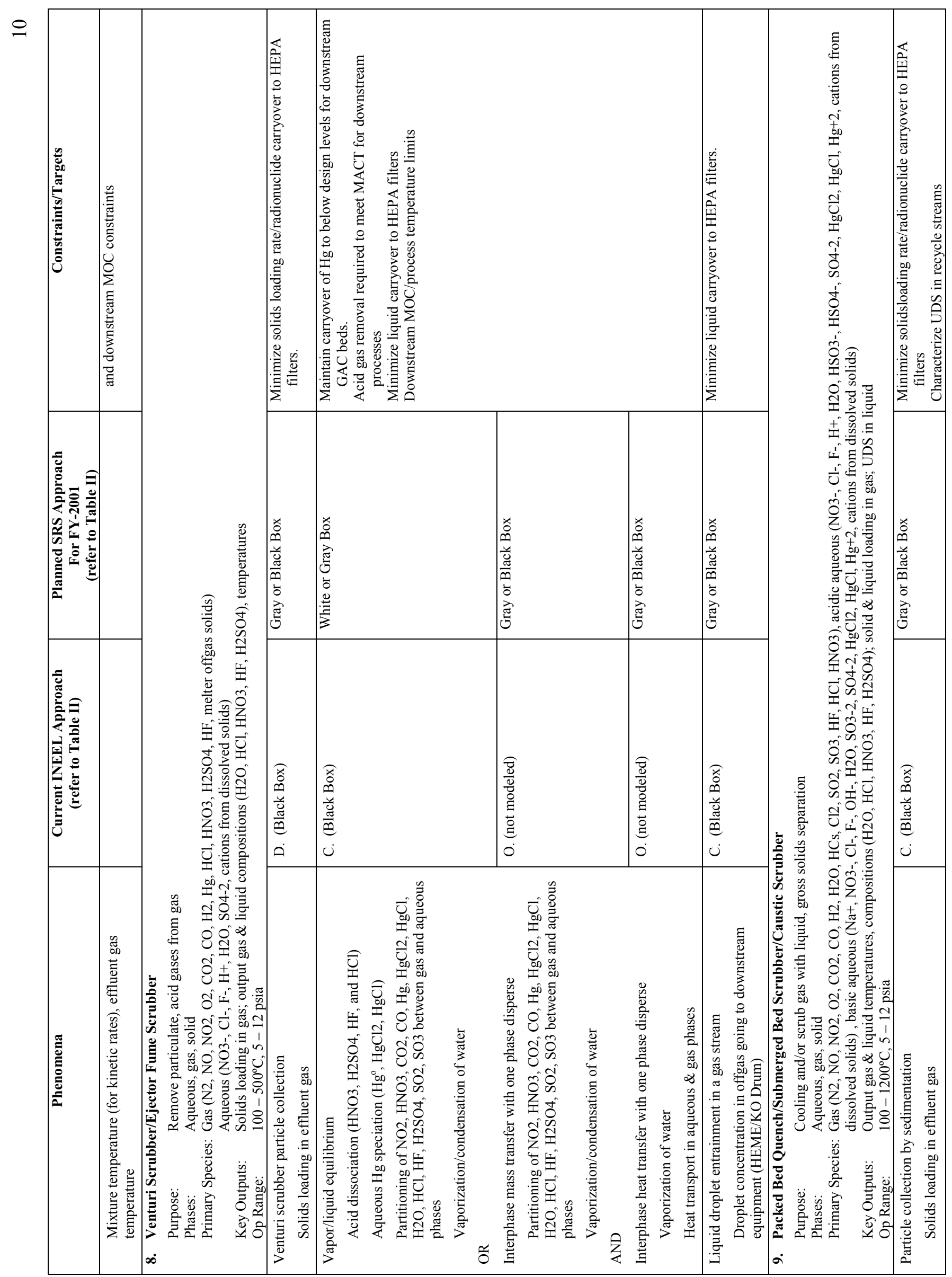




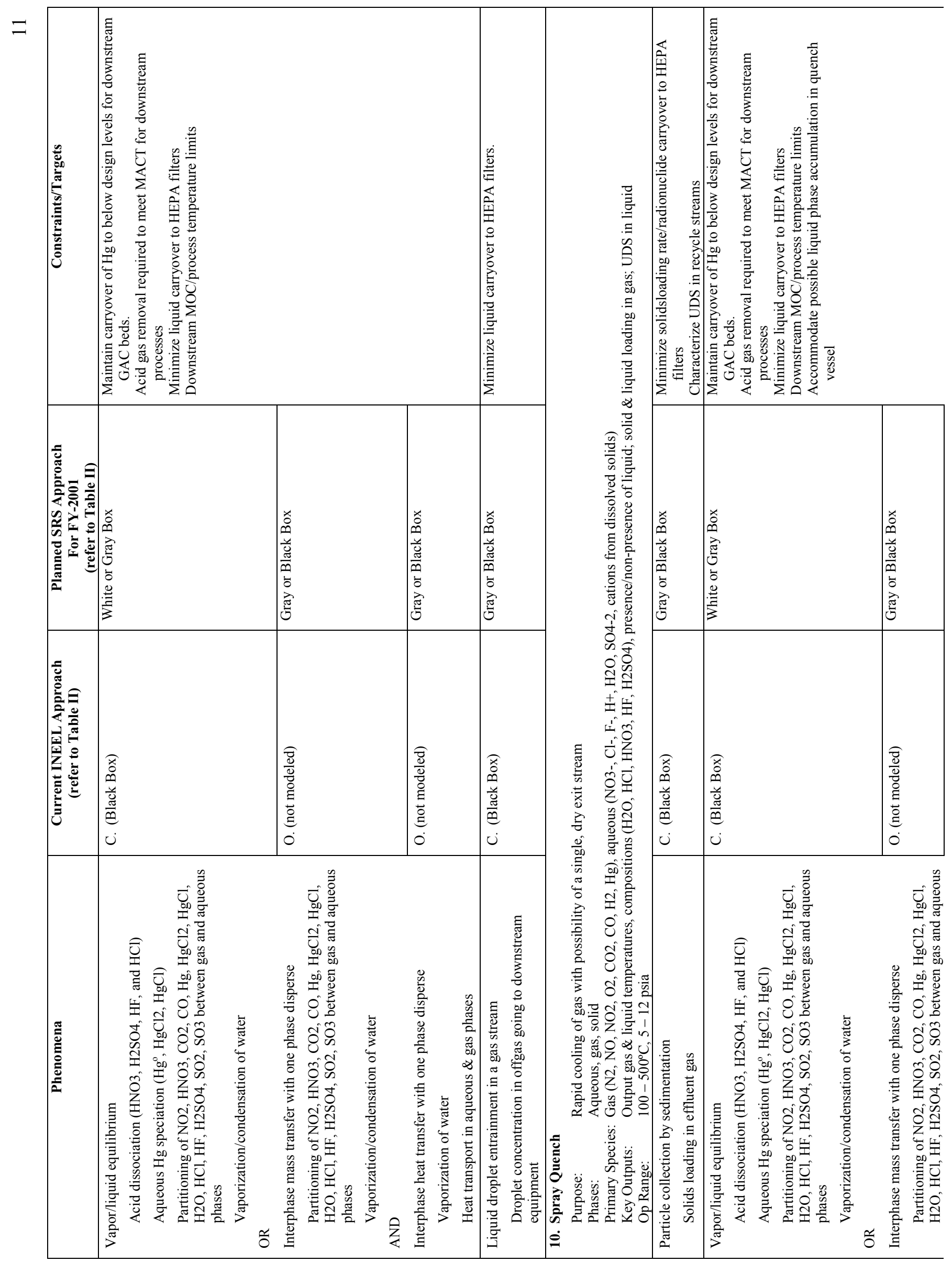




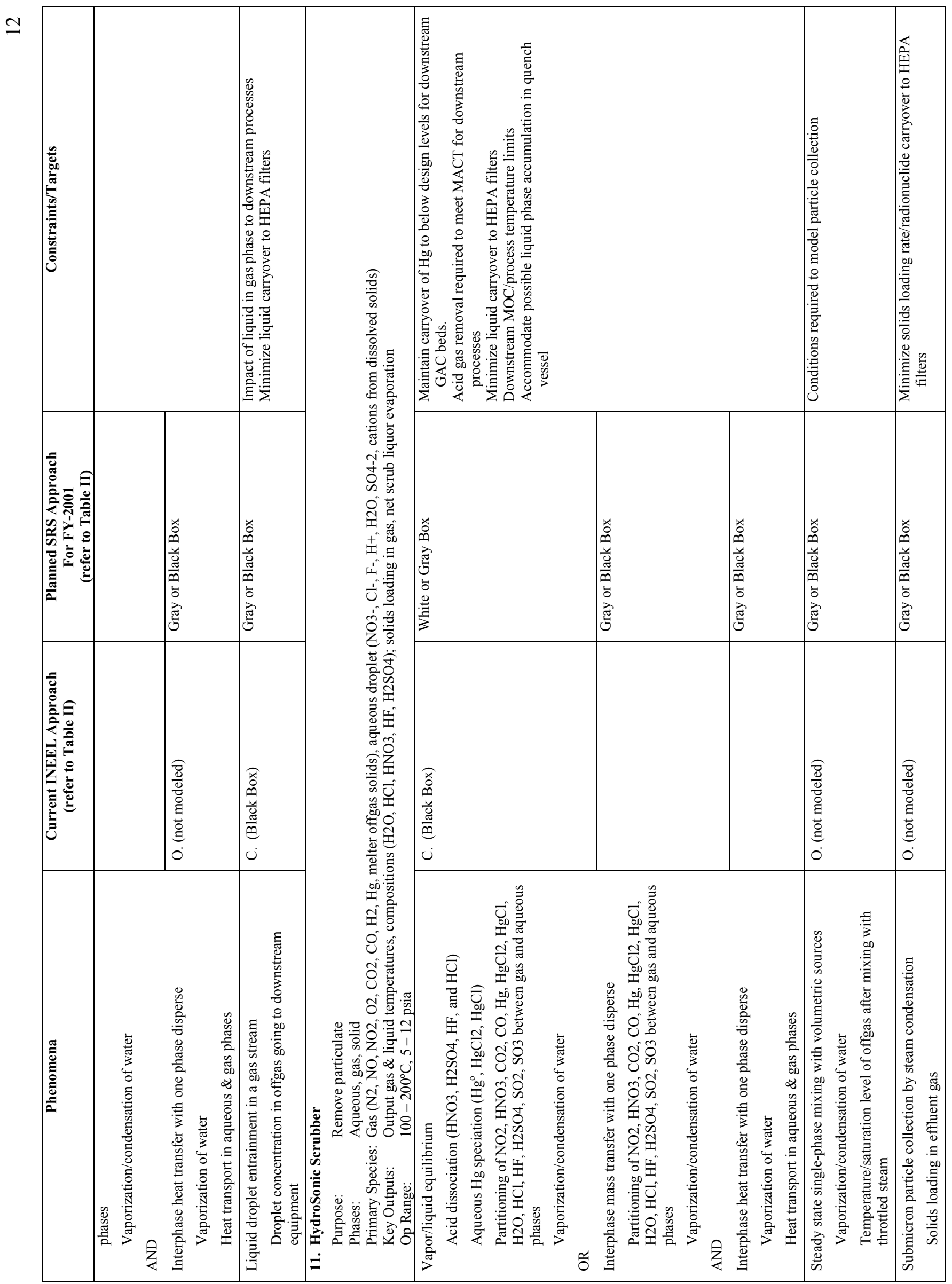




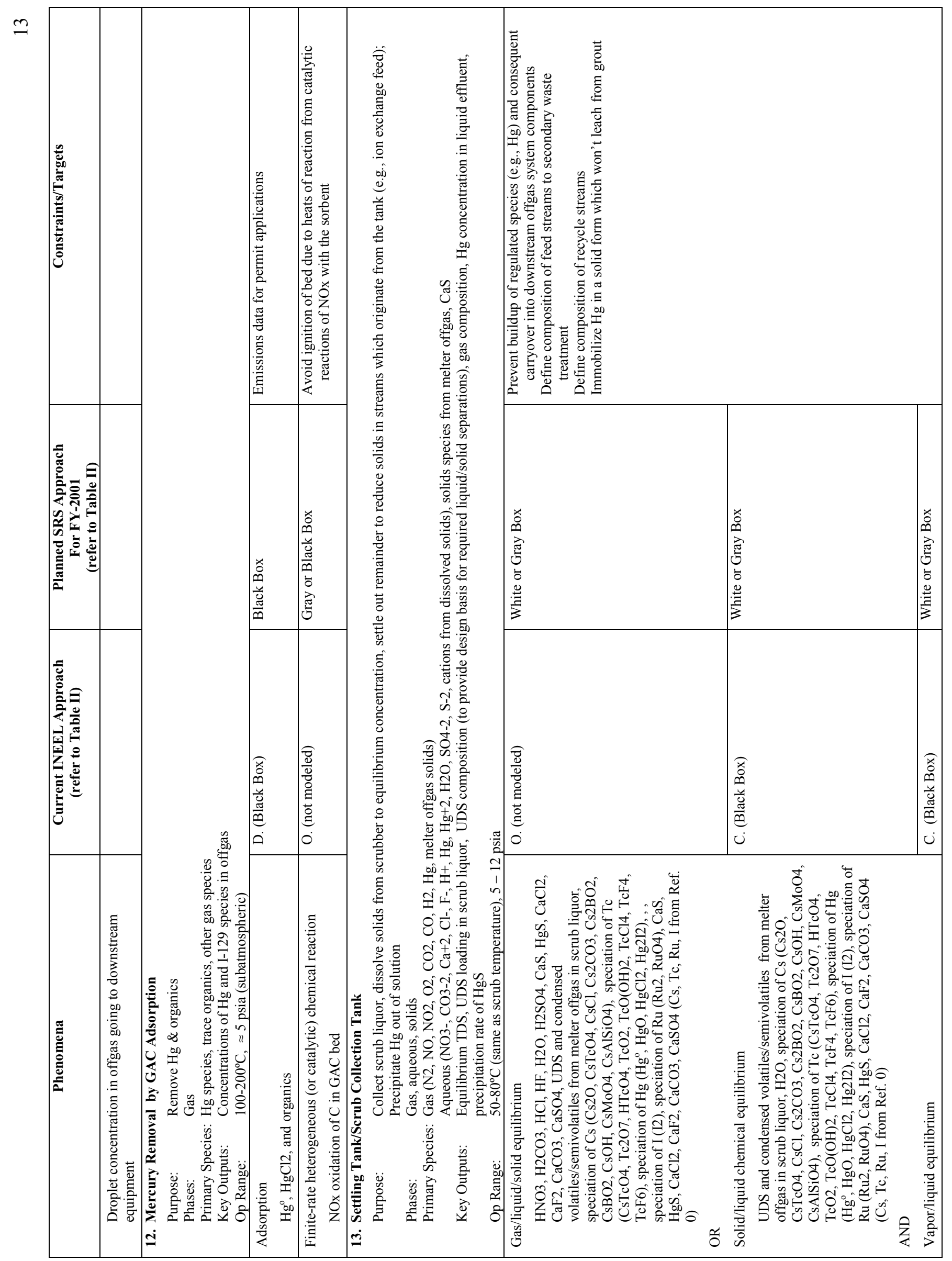




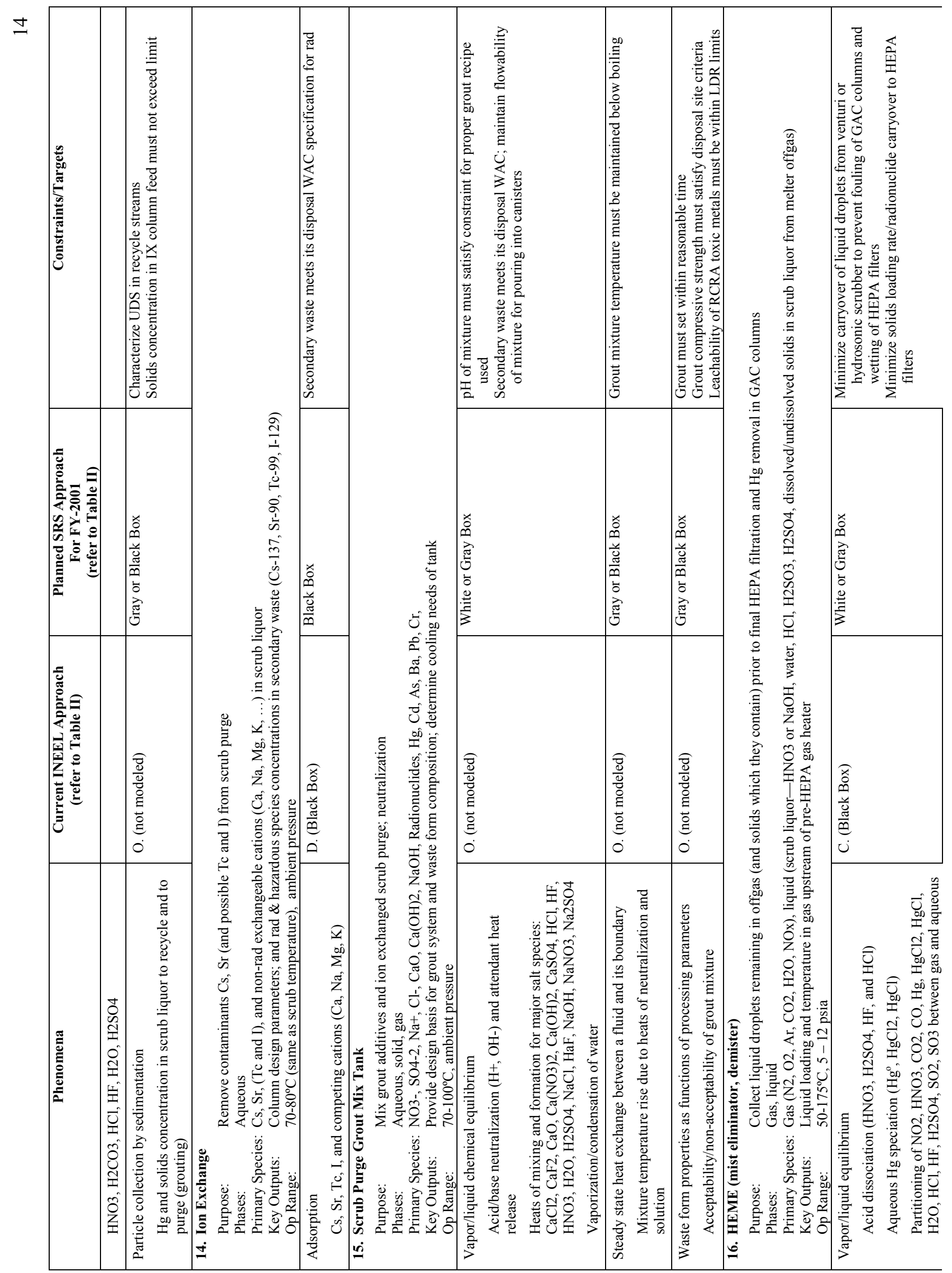




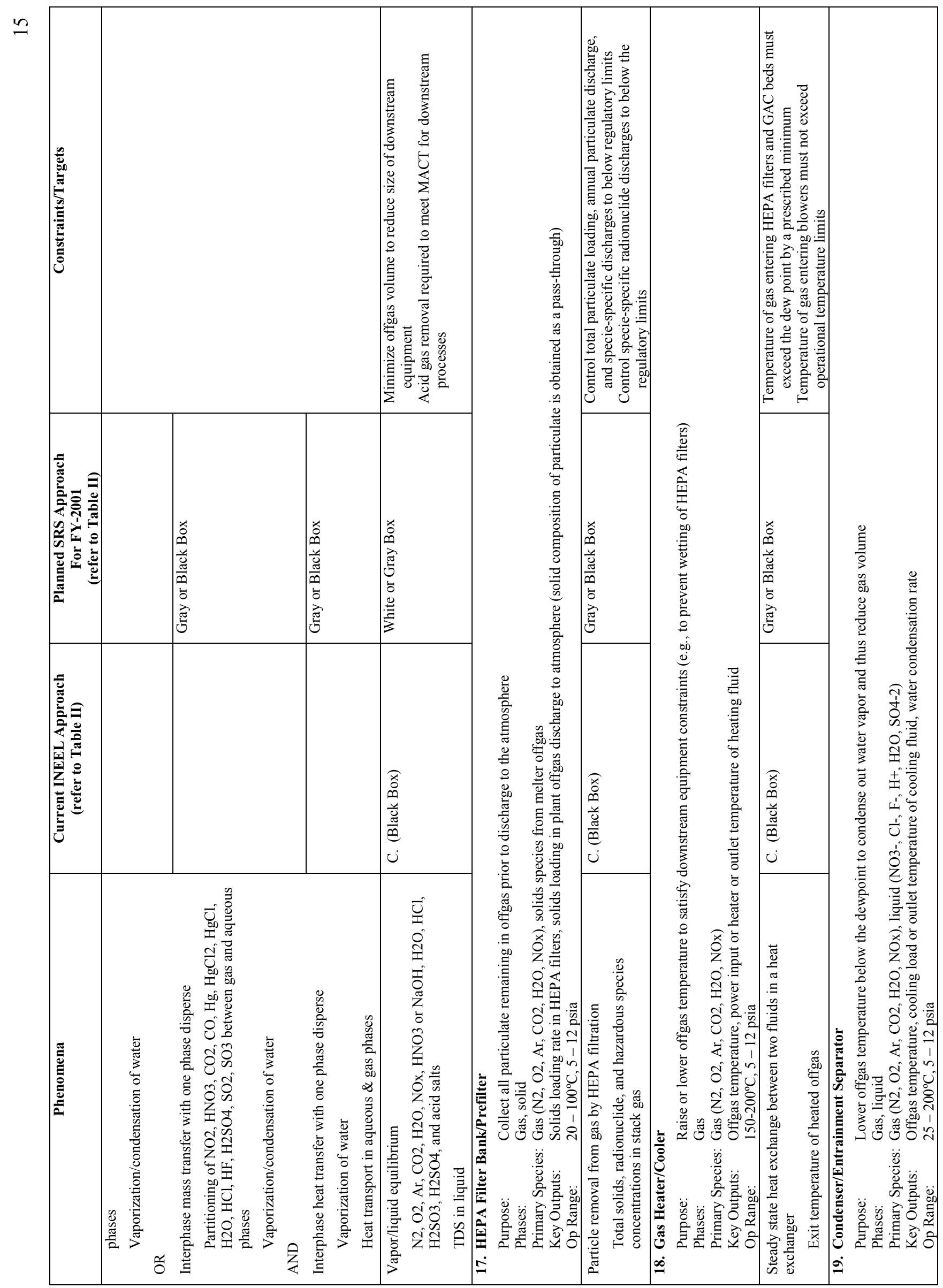




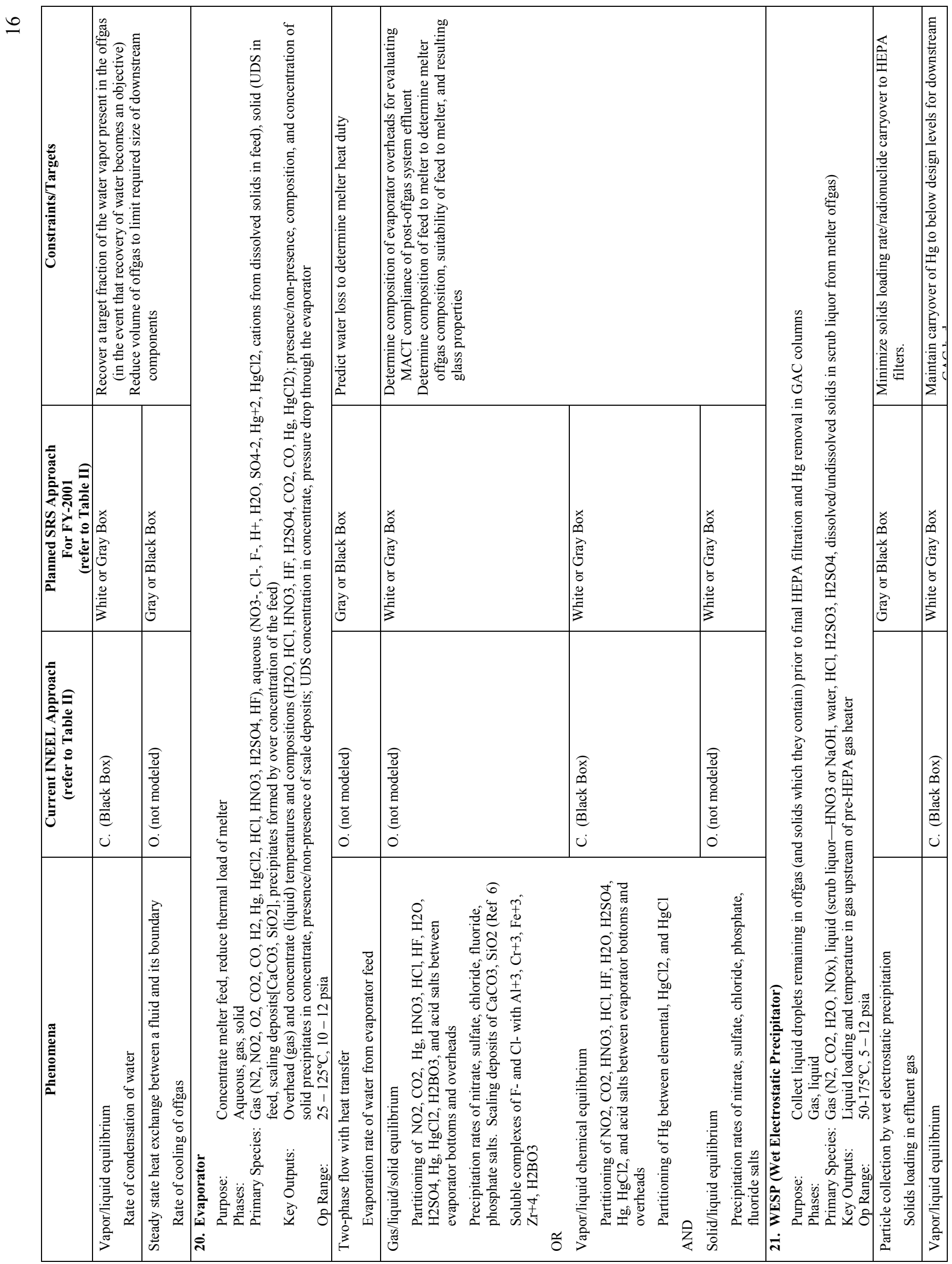




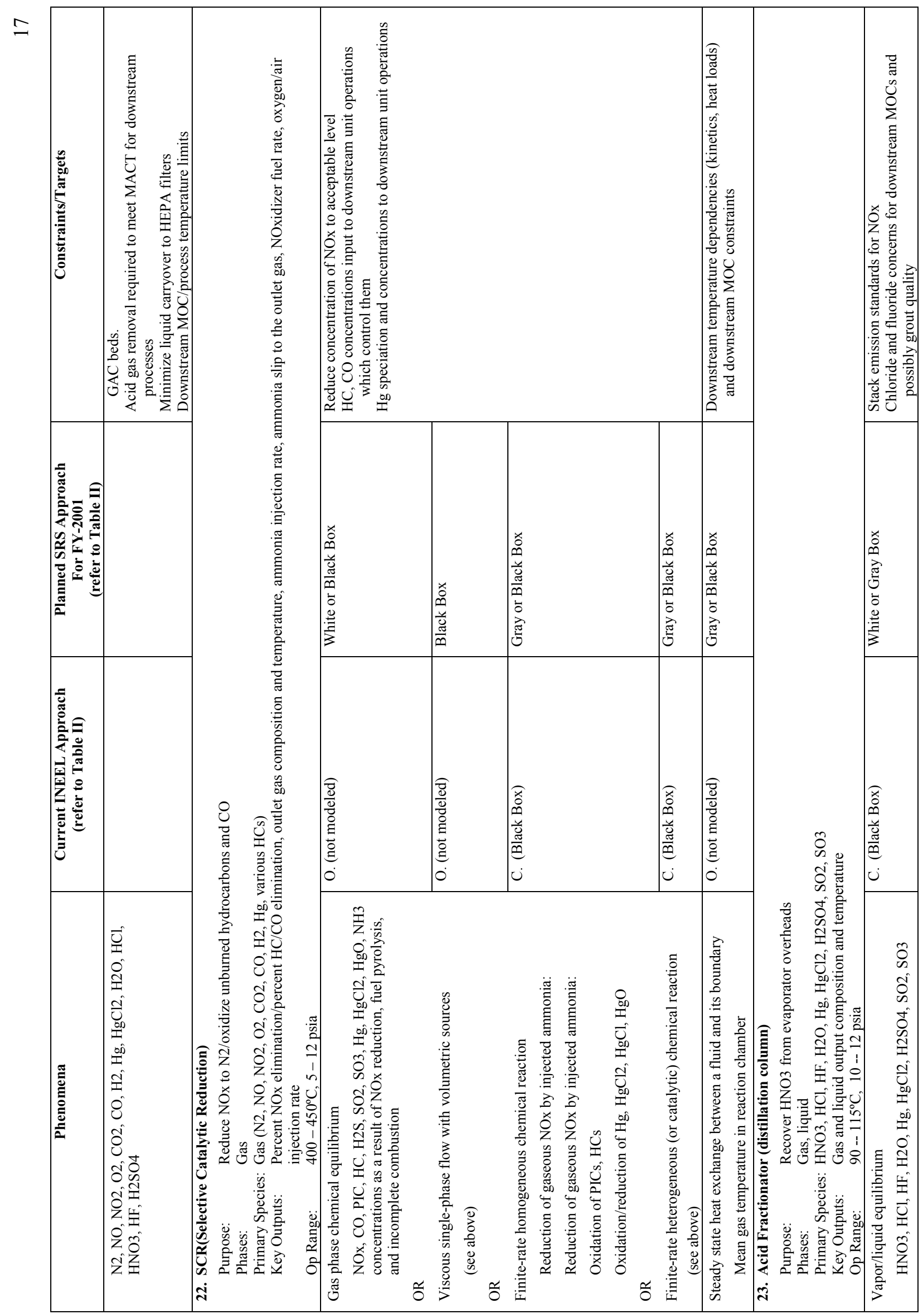



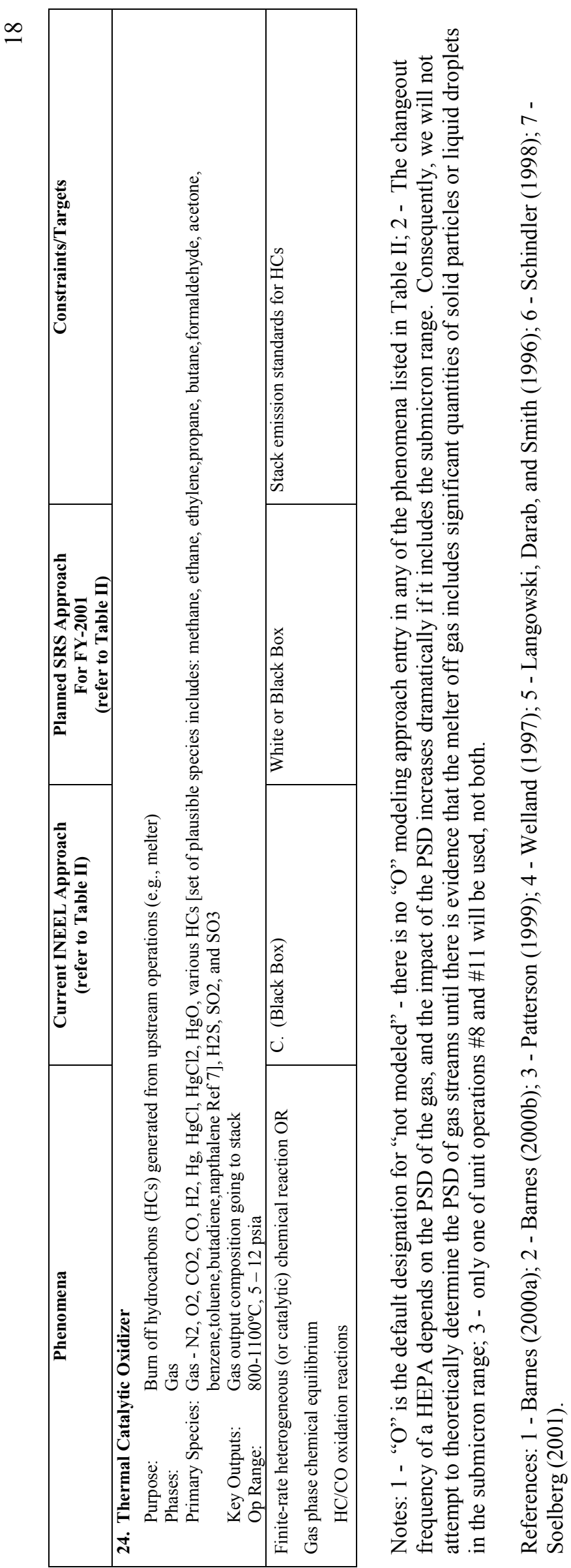
2

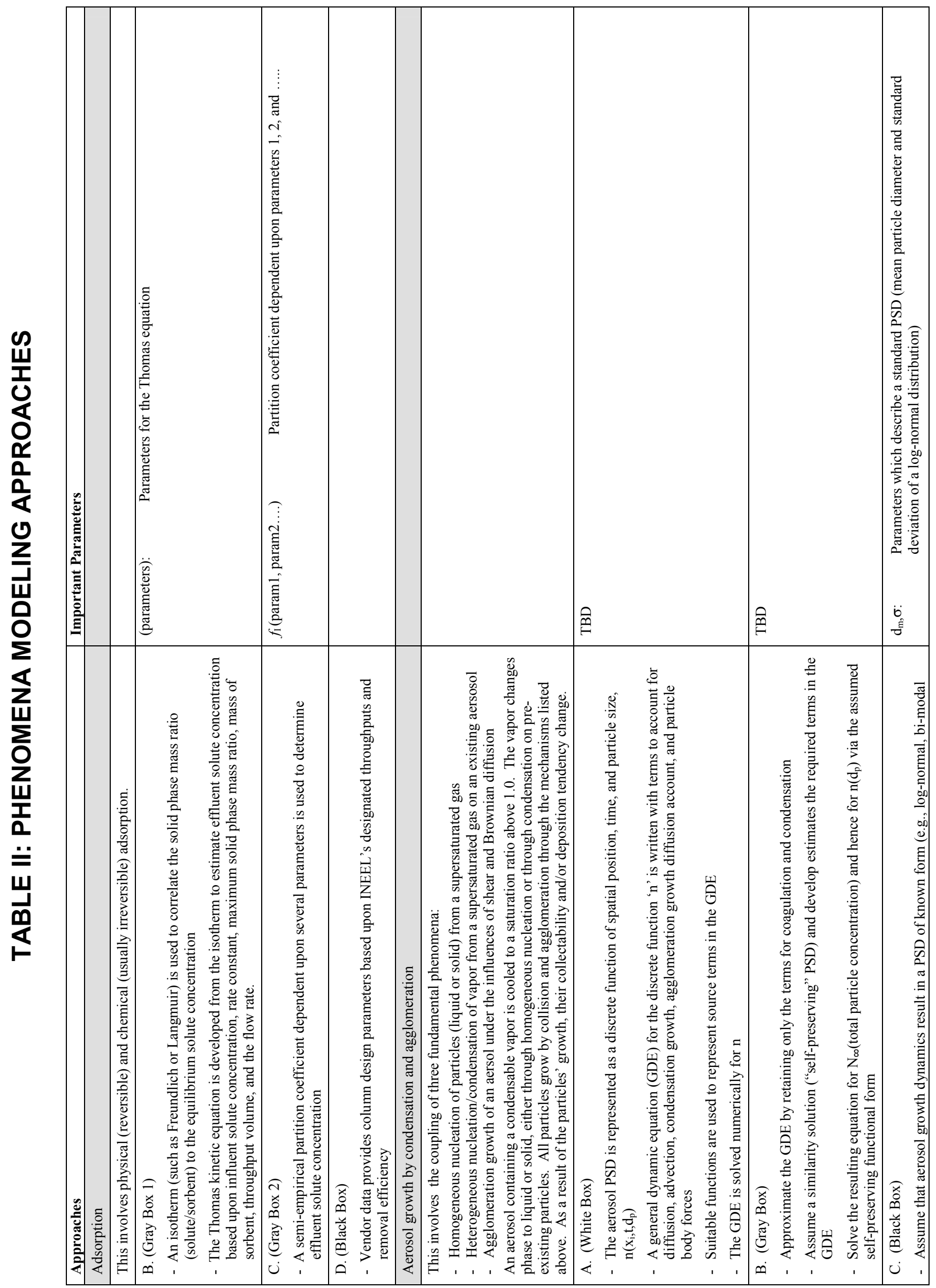




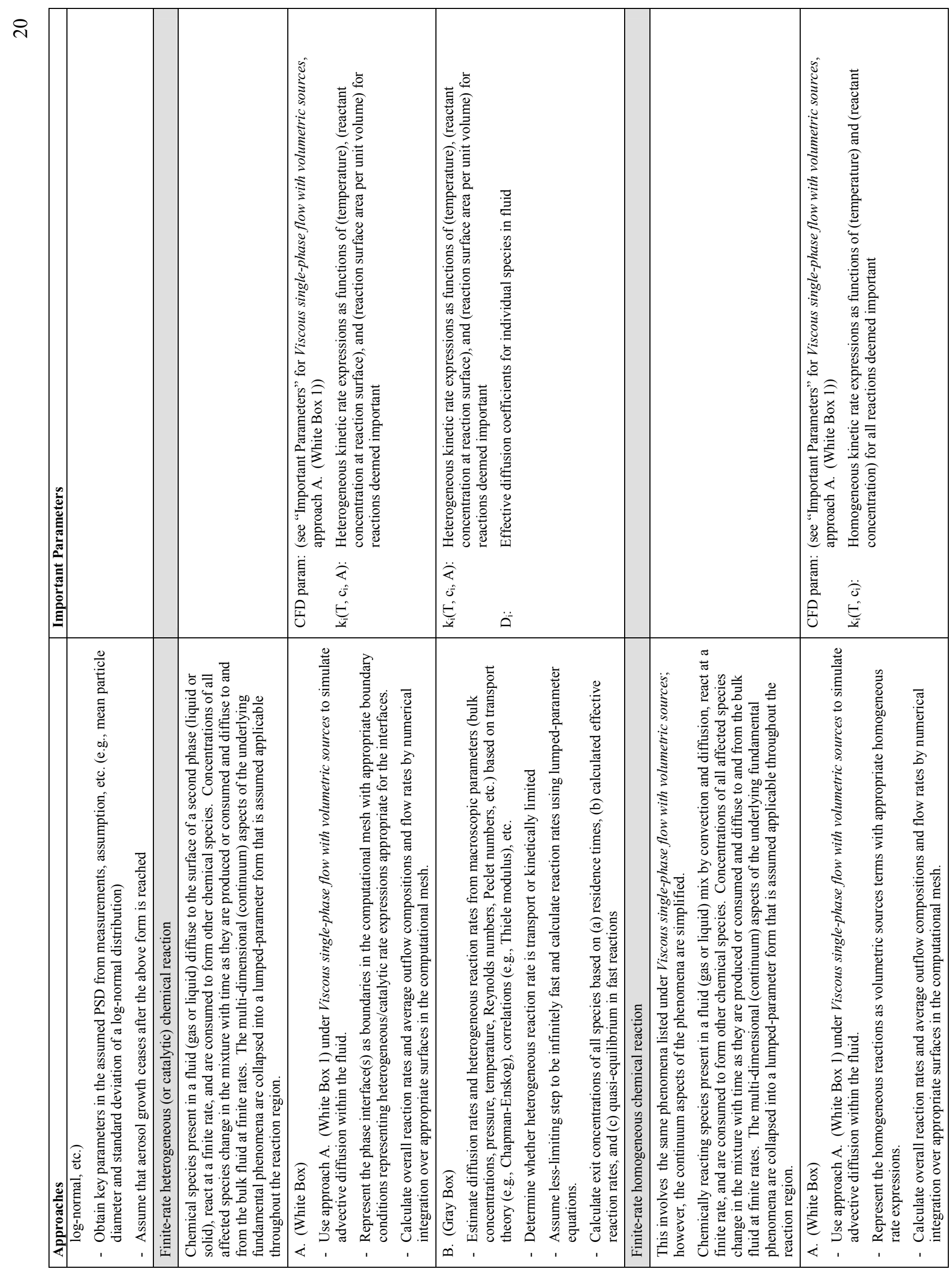




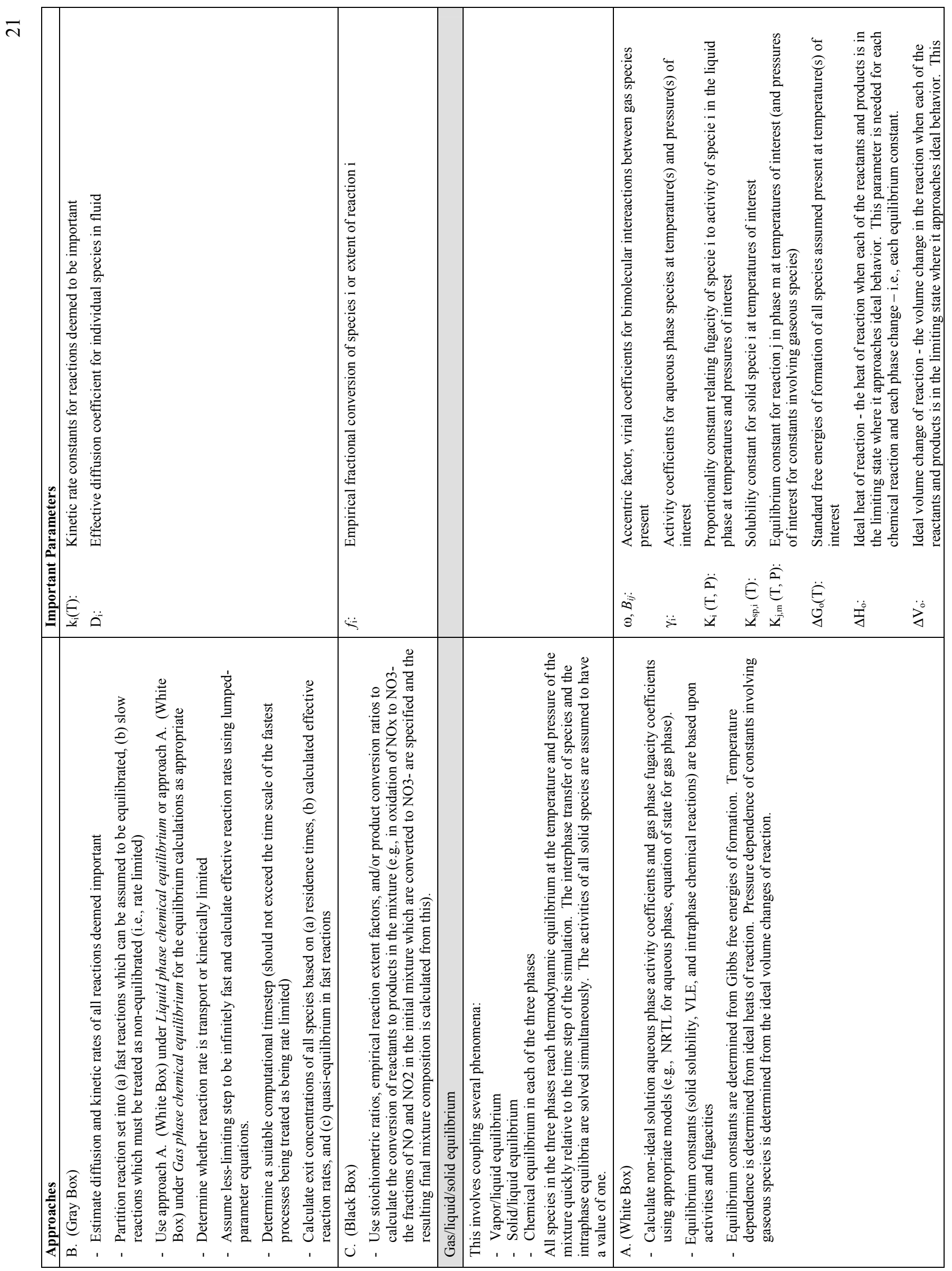




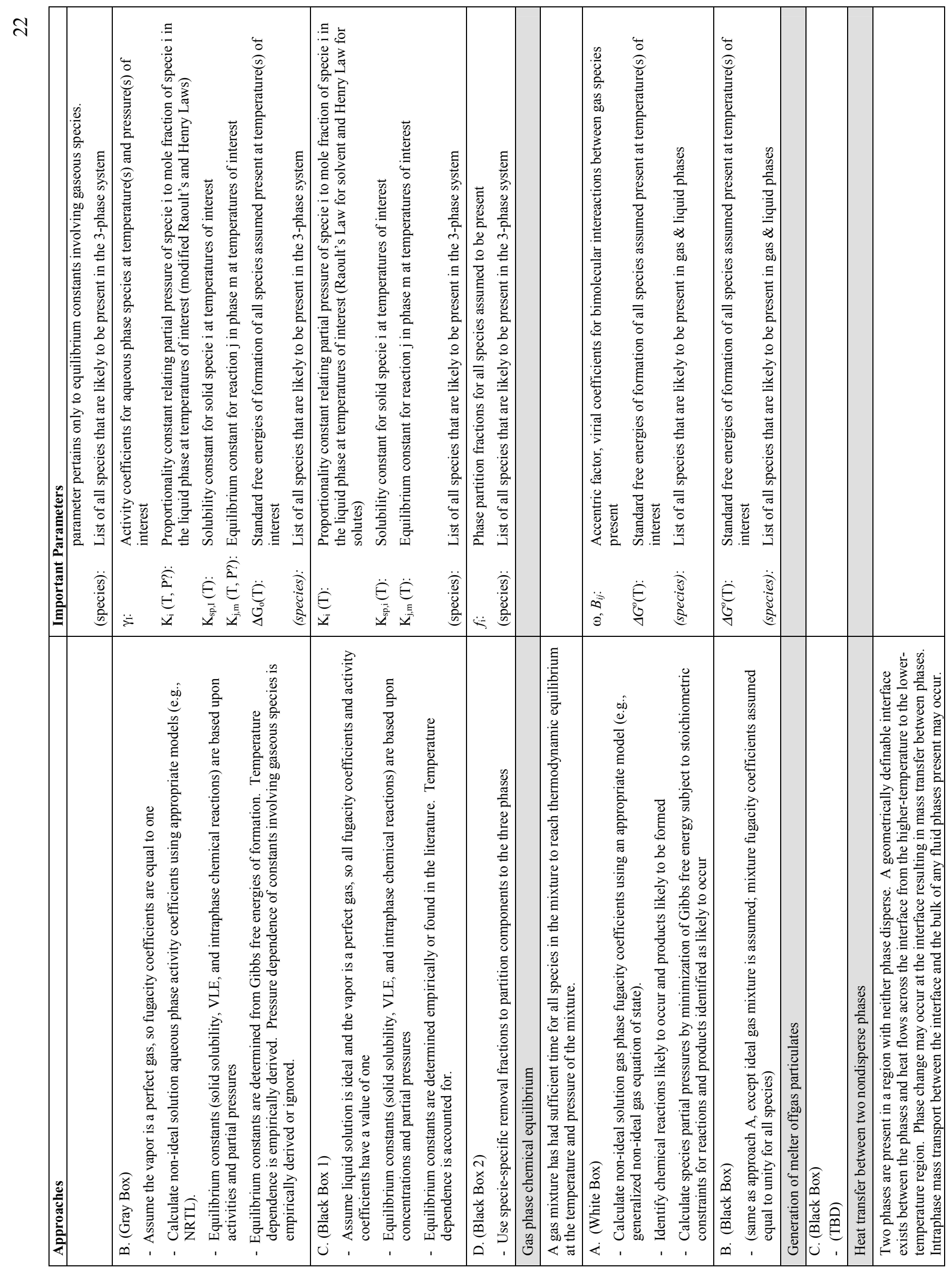




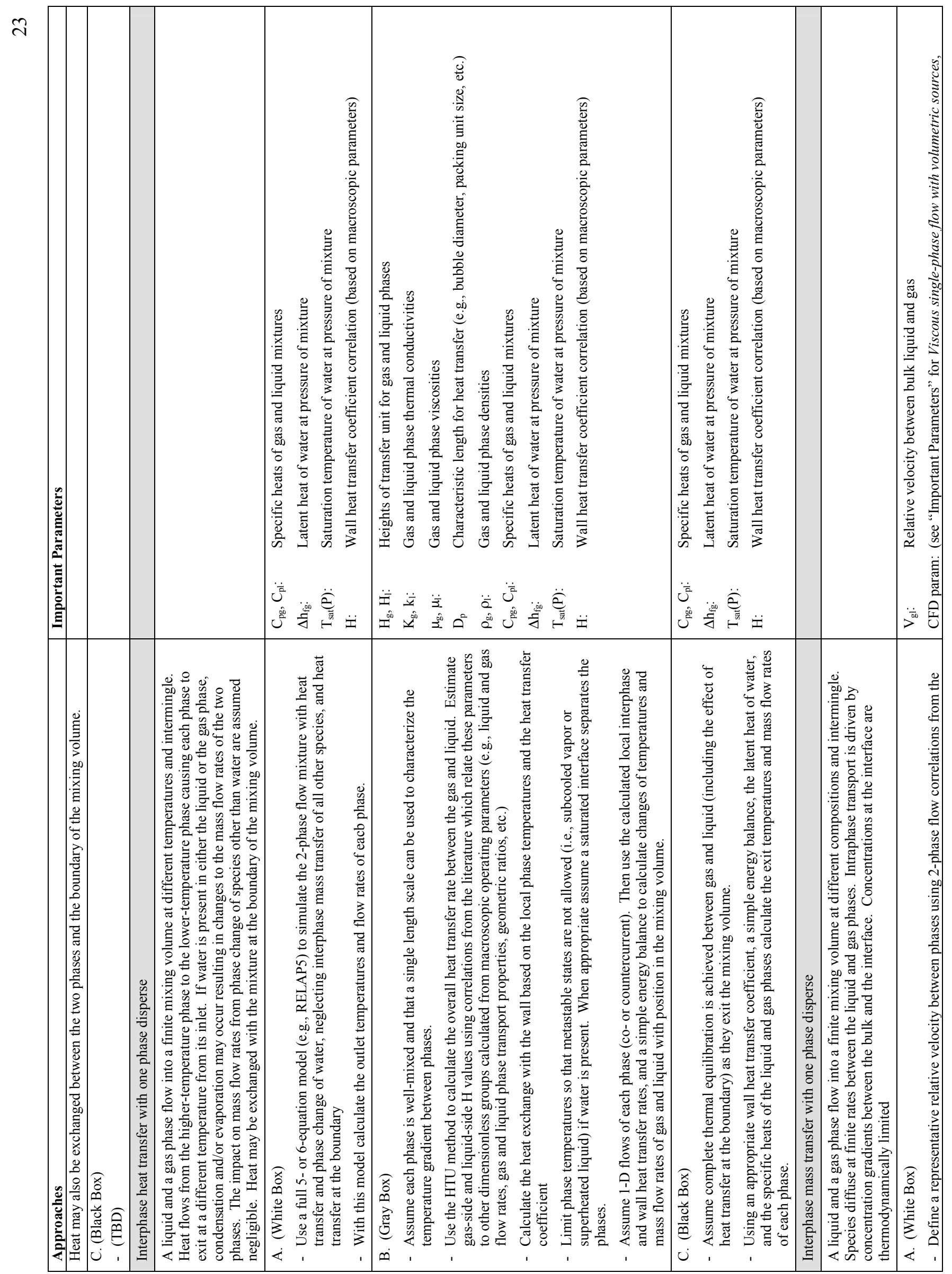




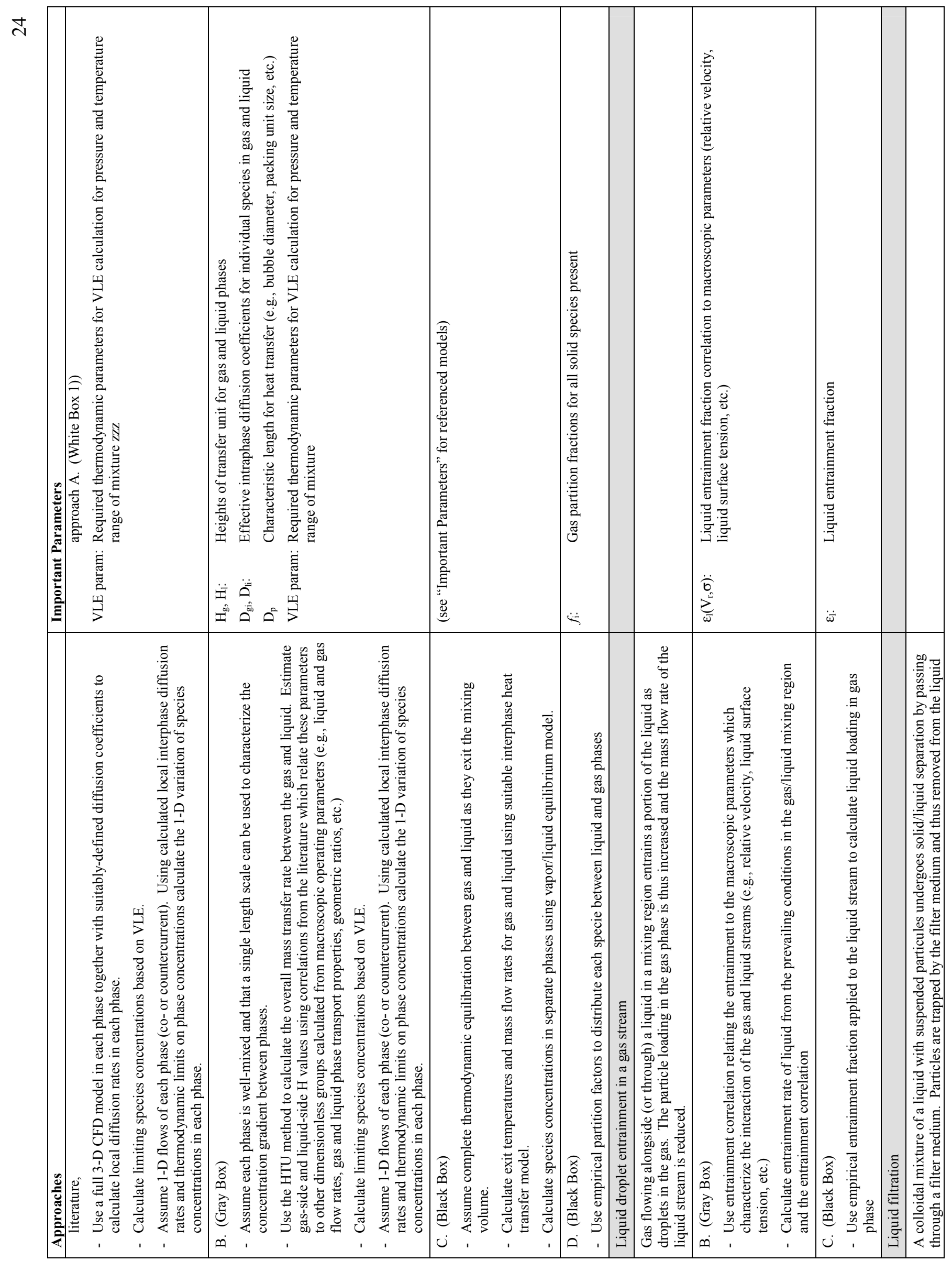




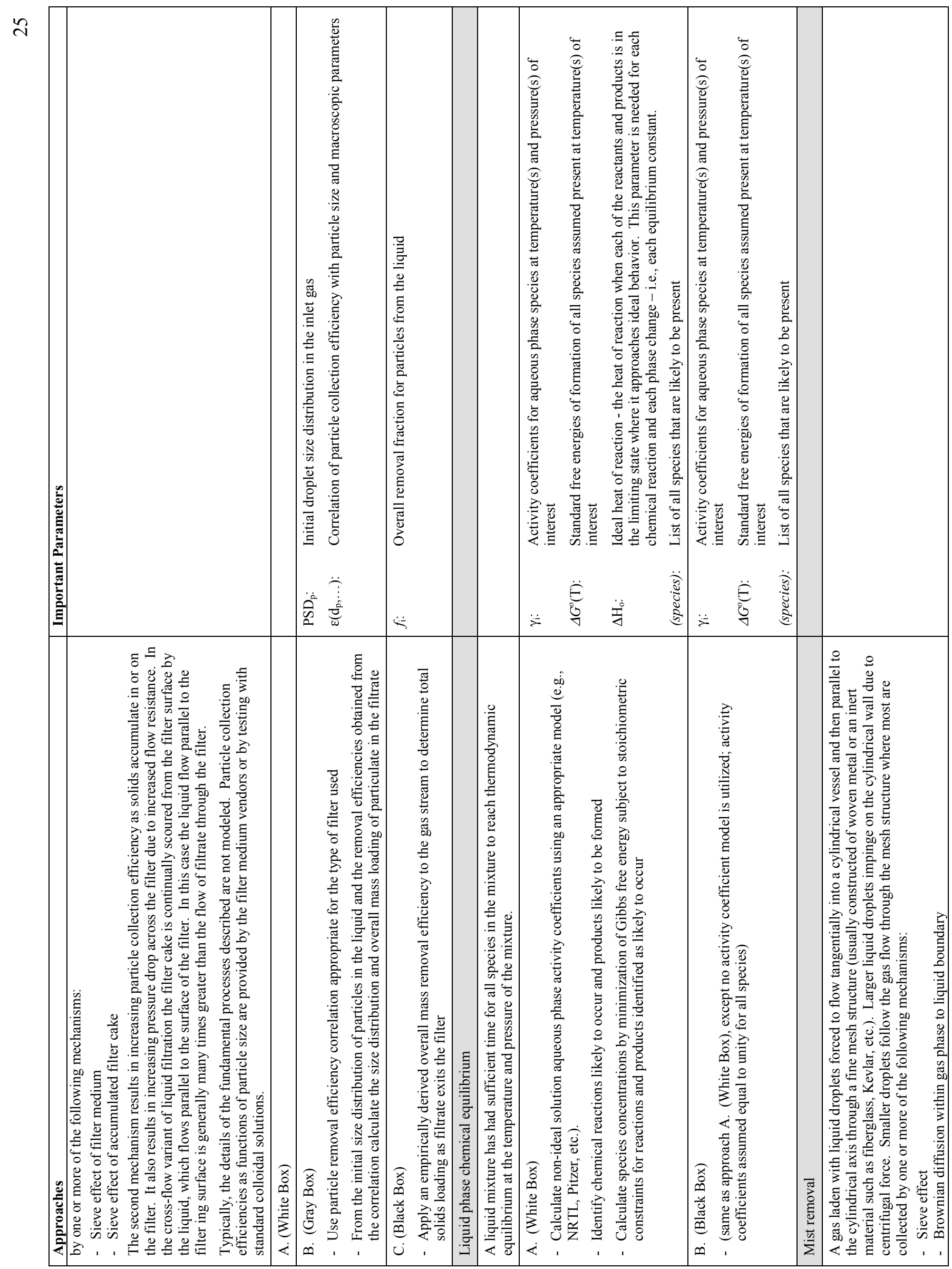




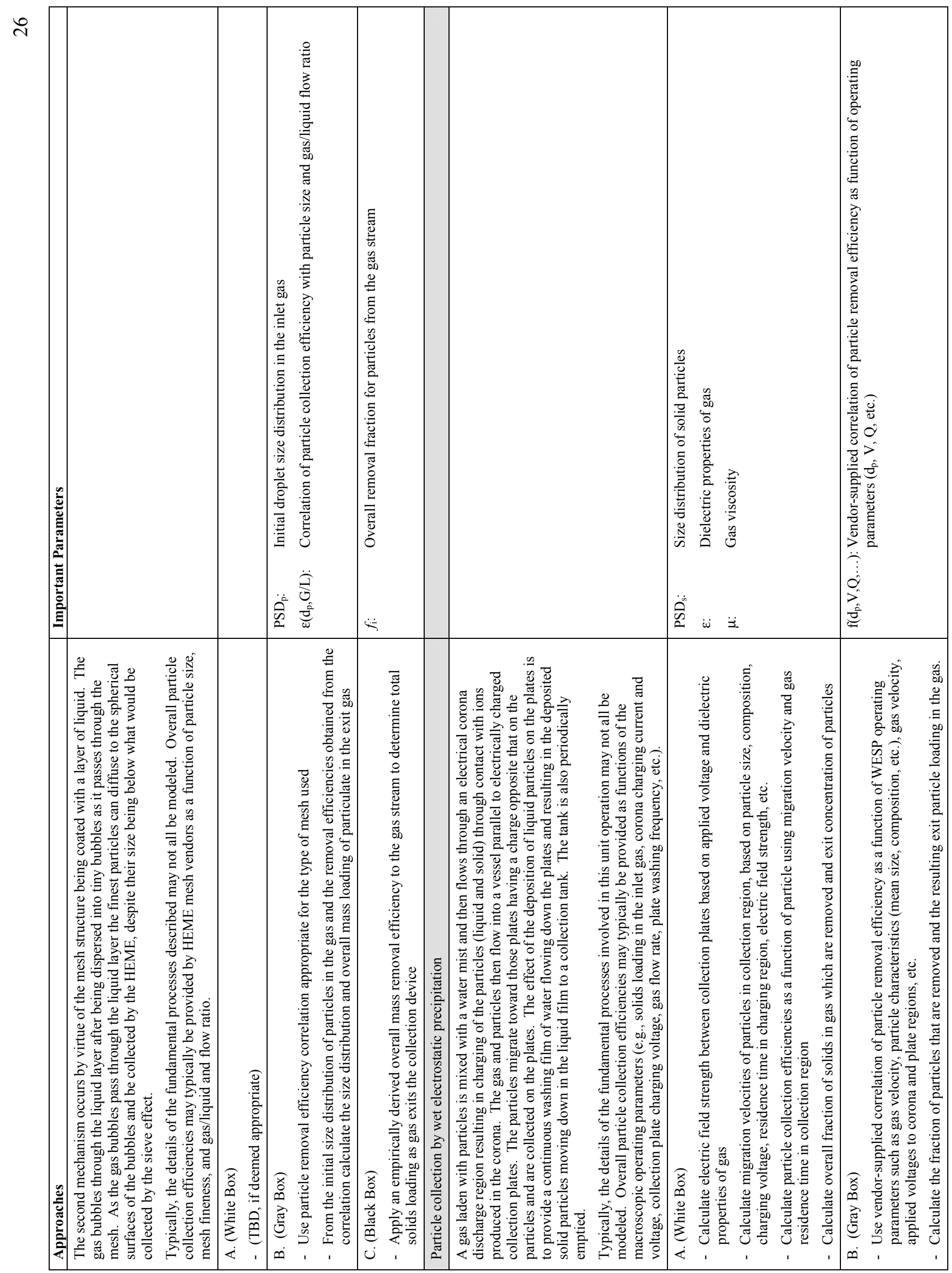




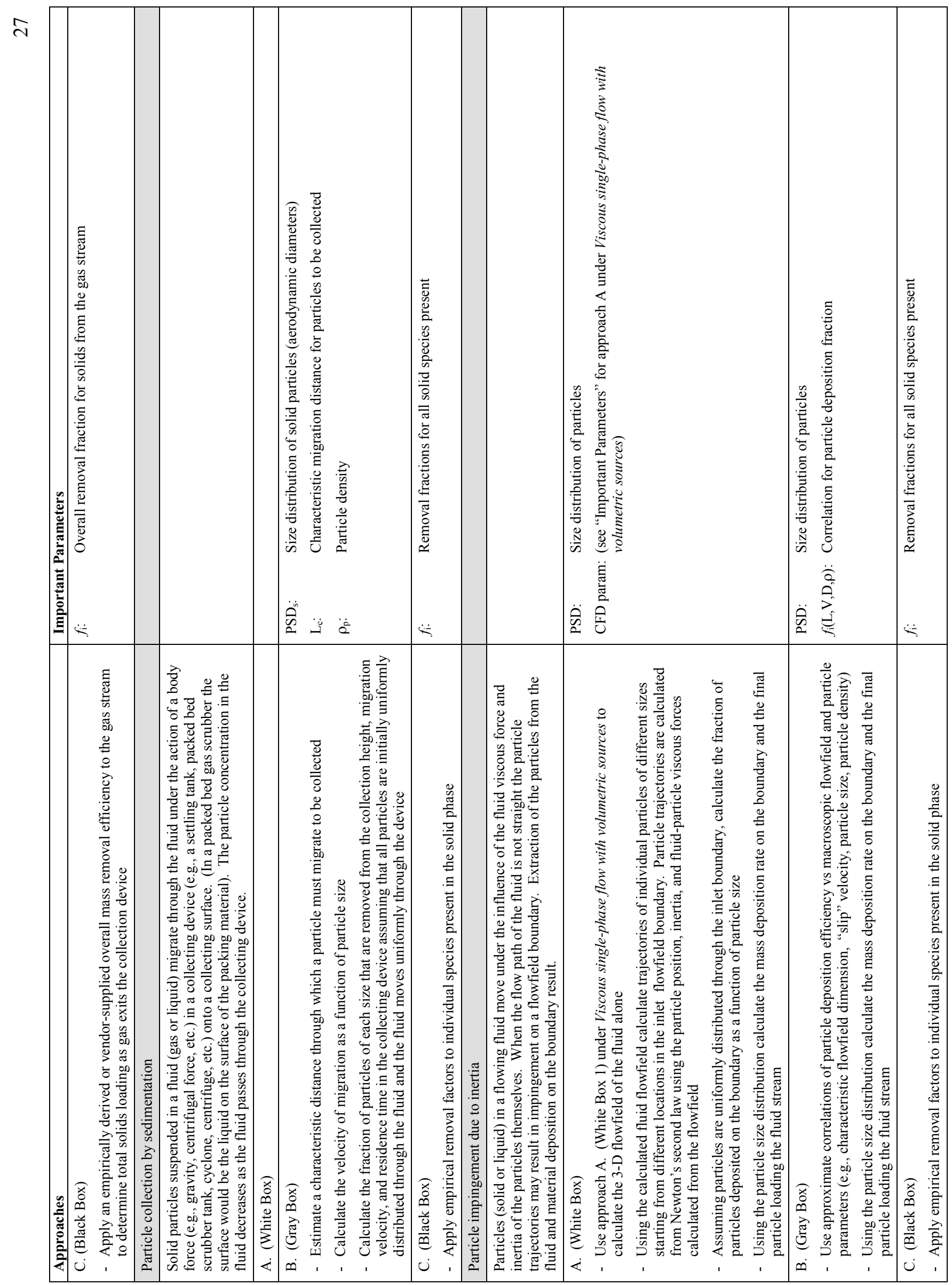




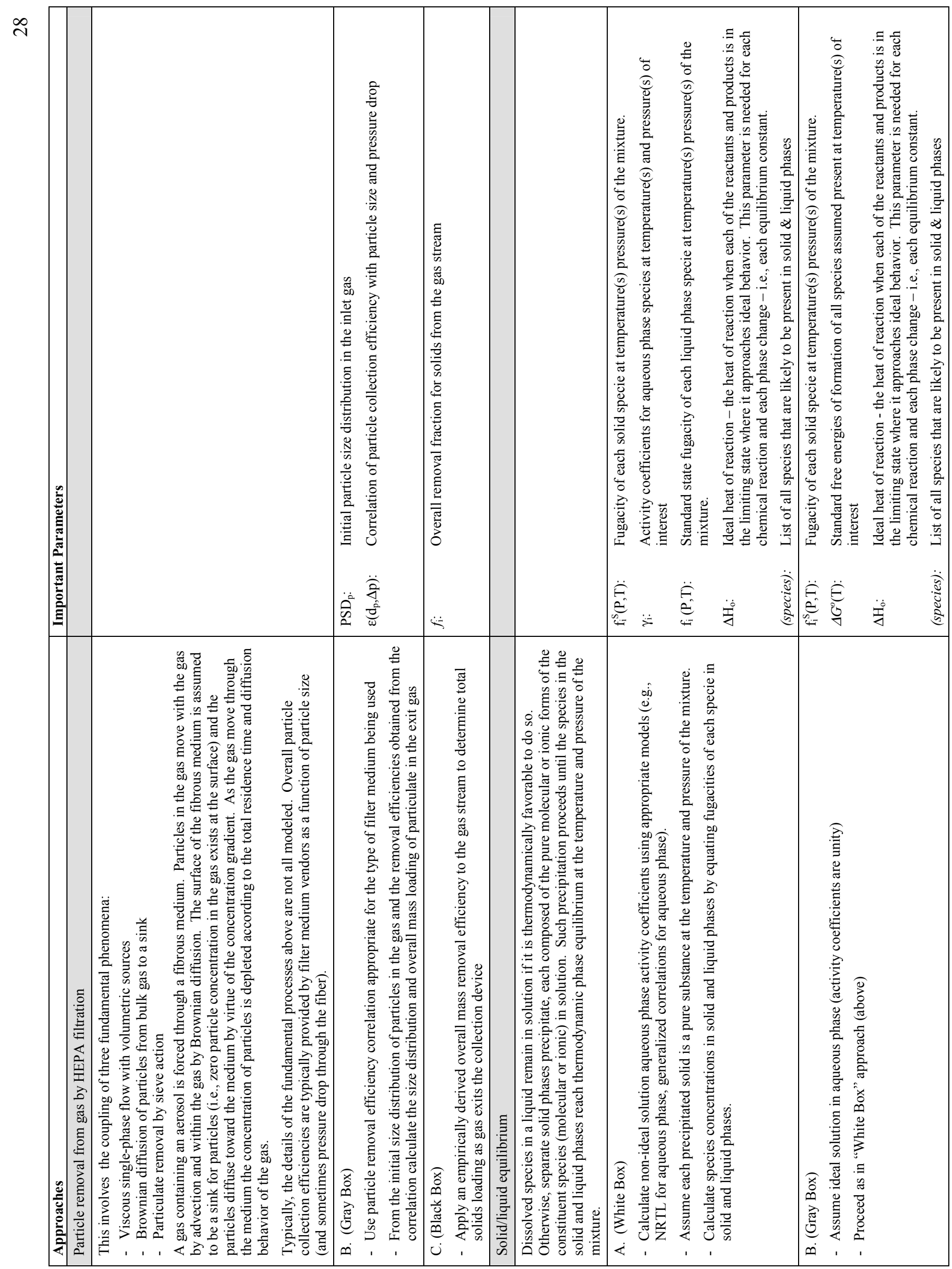




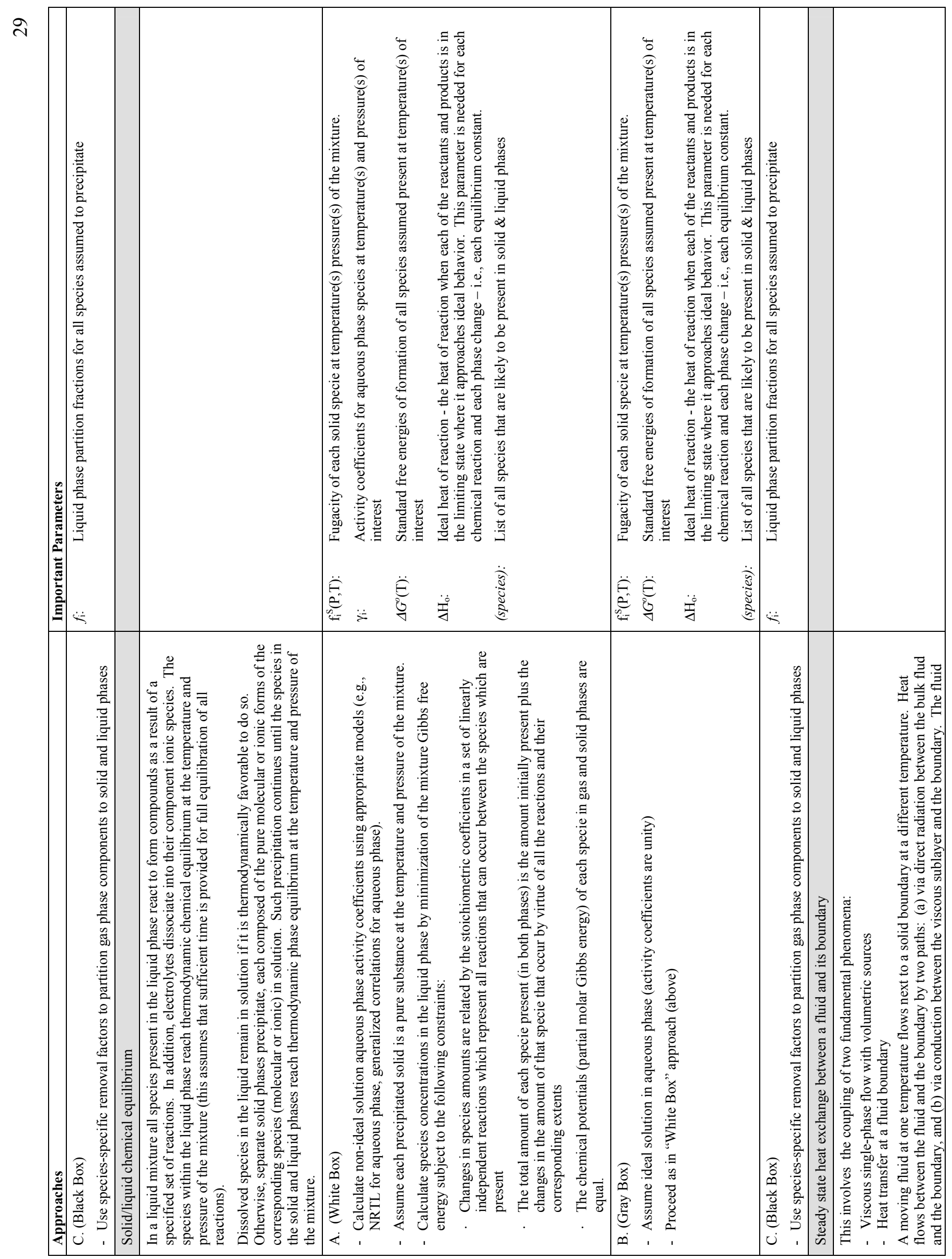




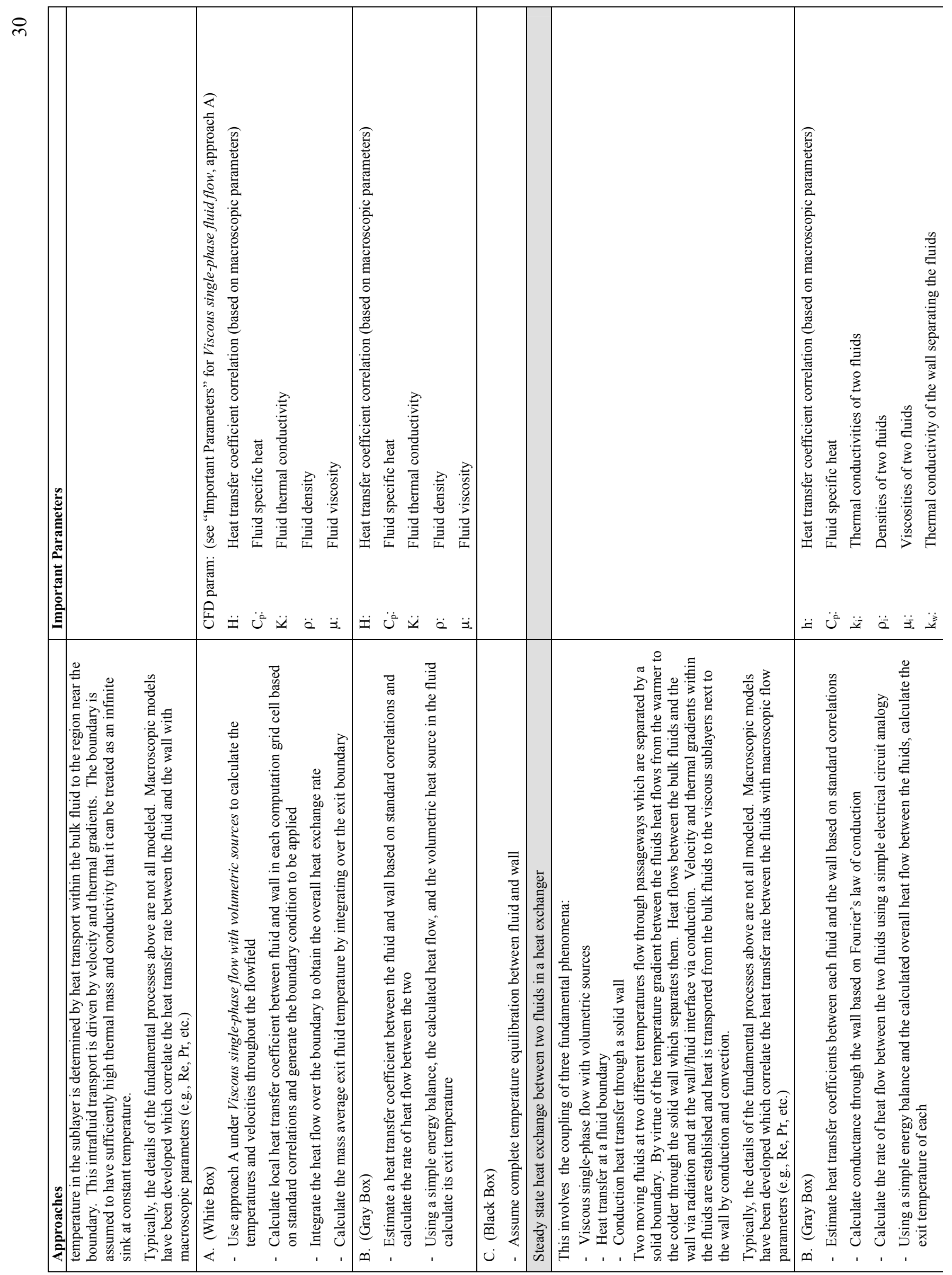




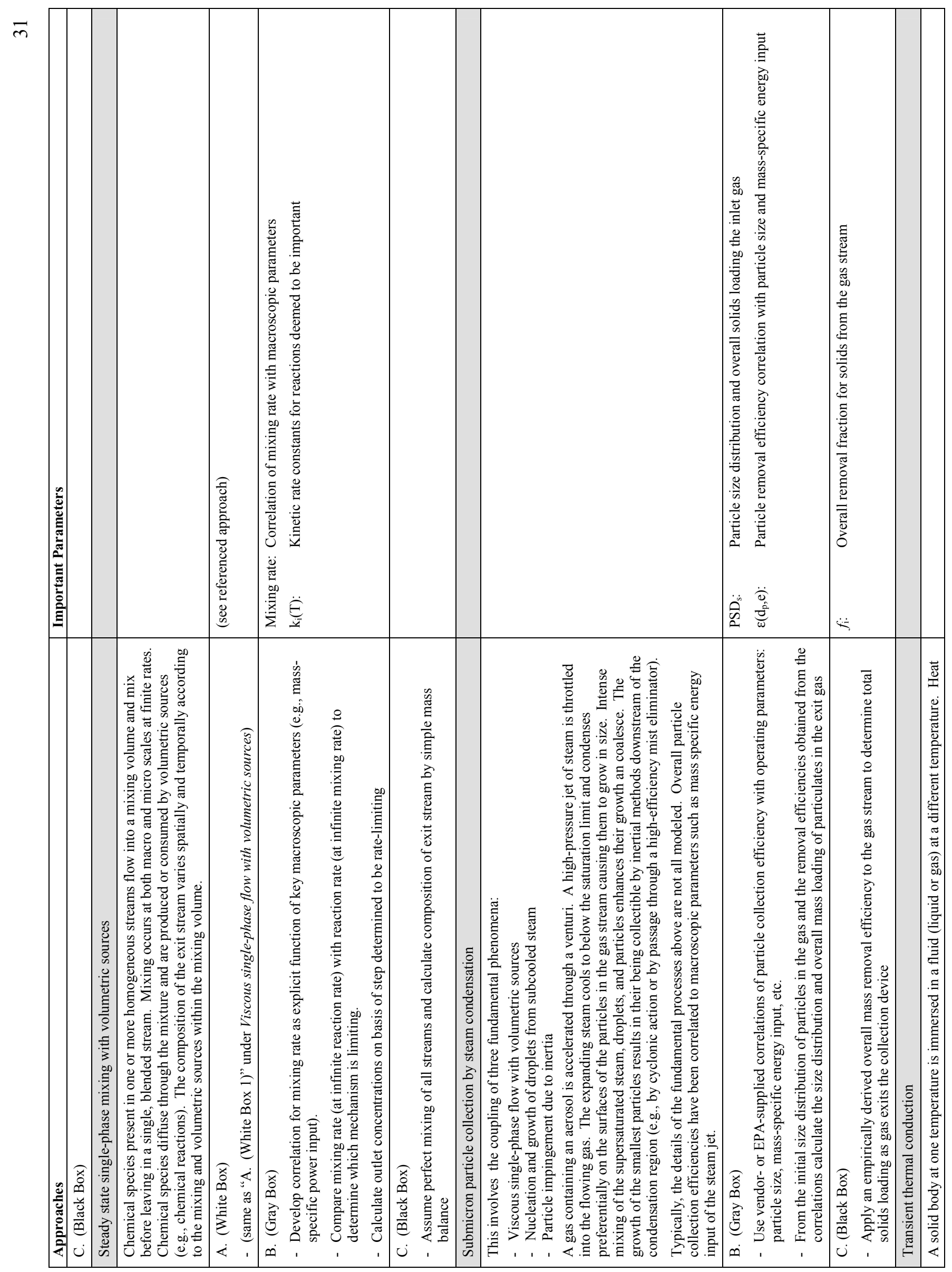




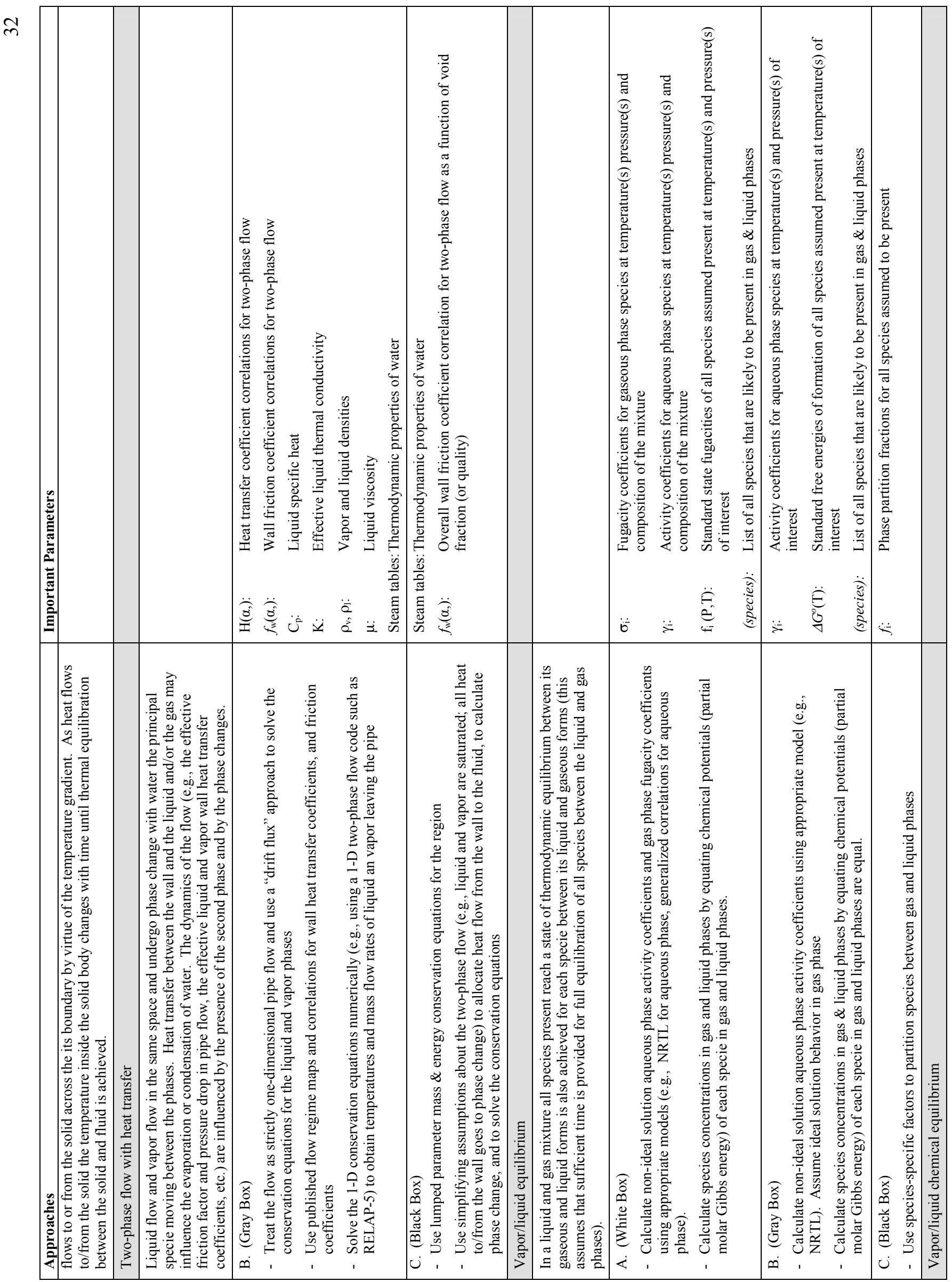




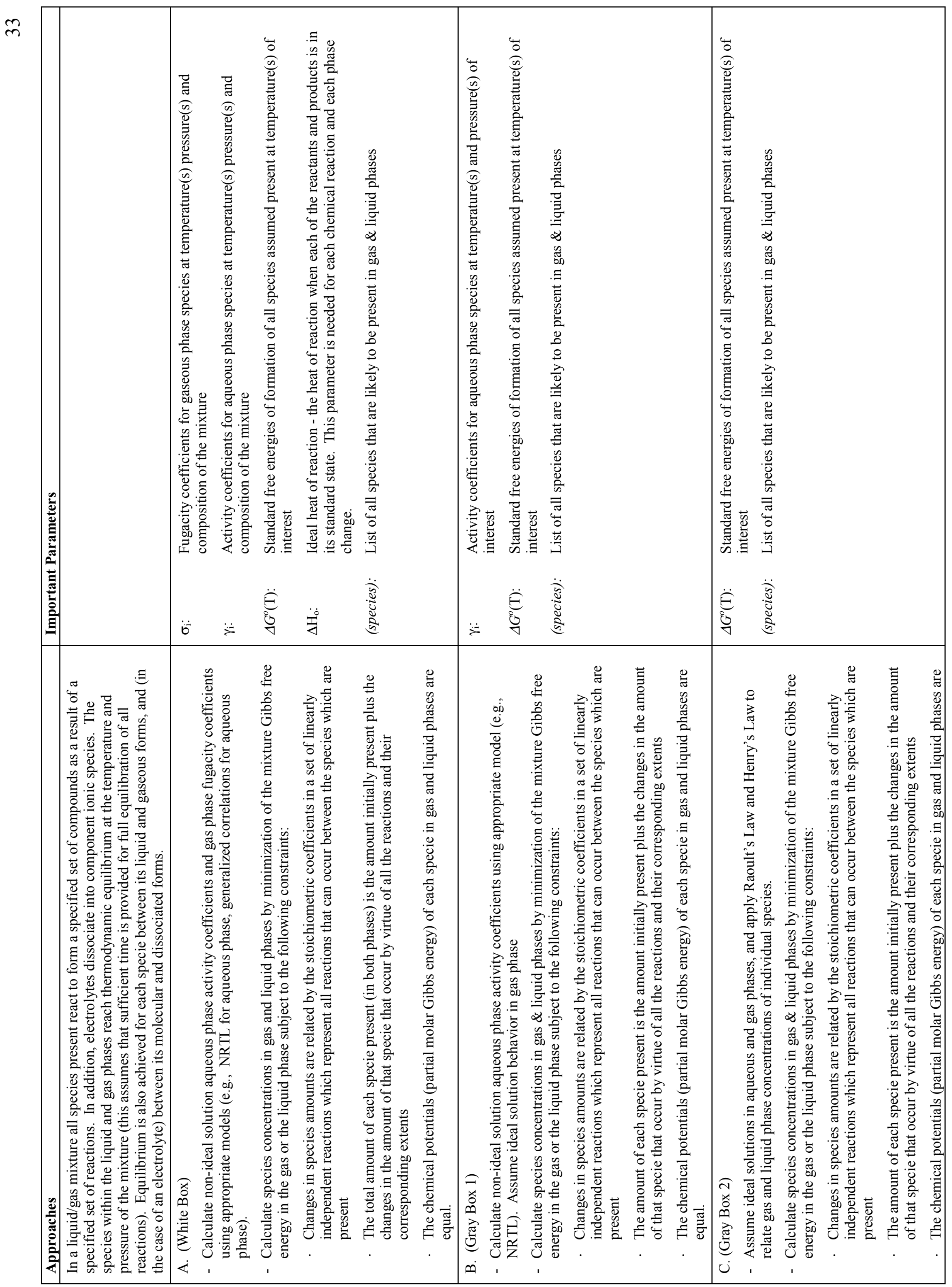




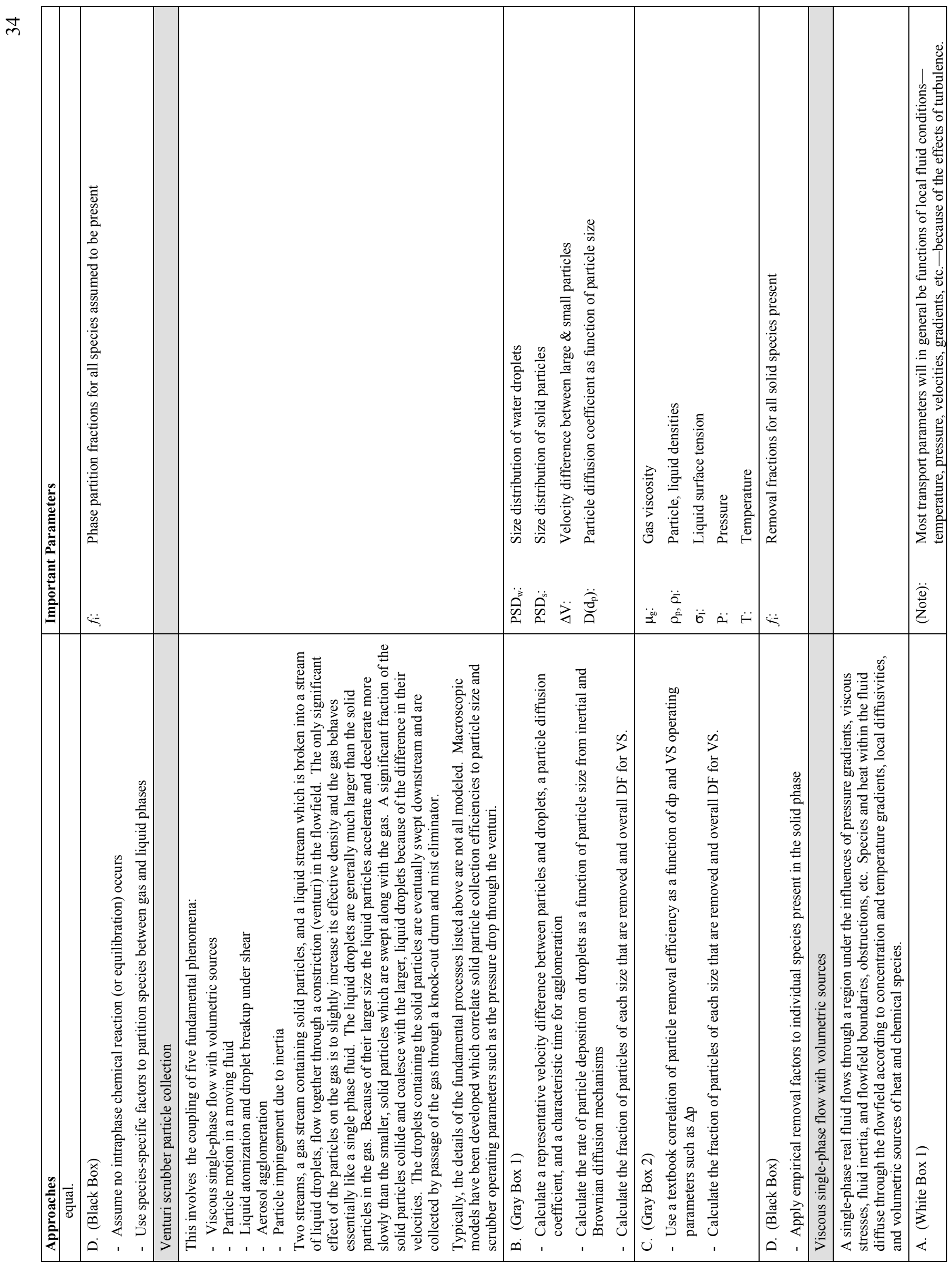



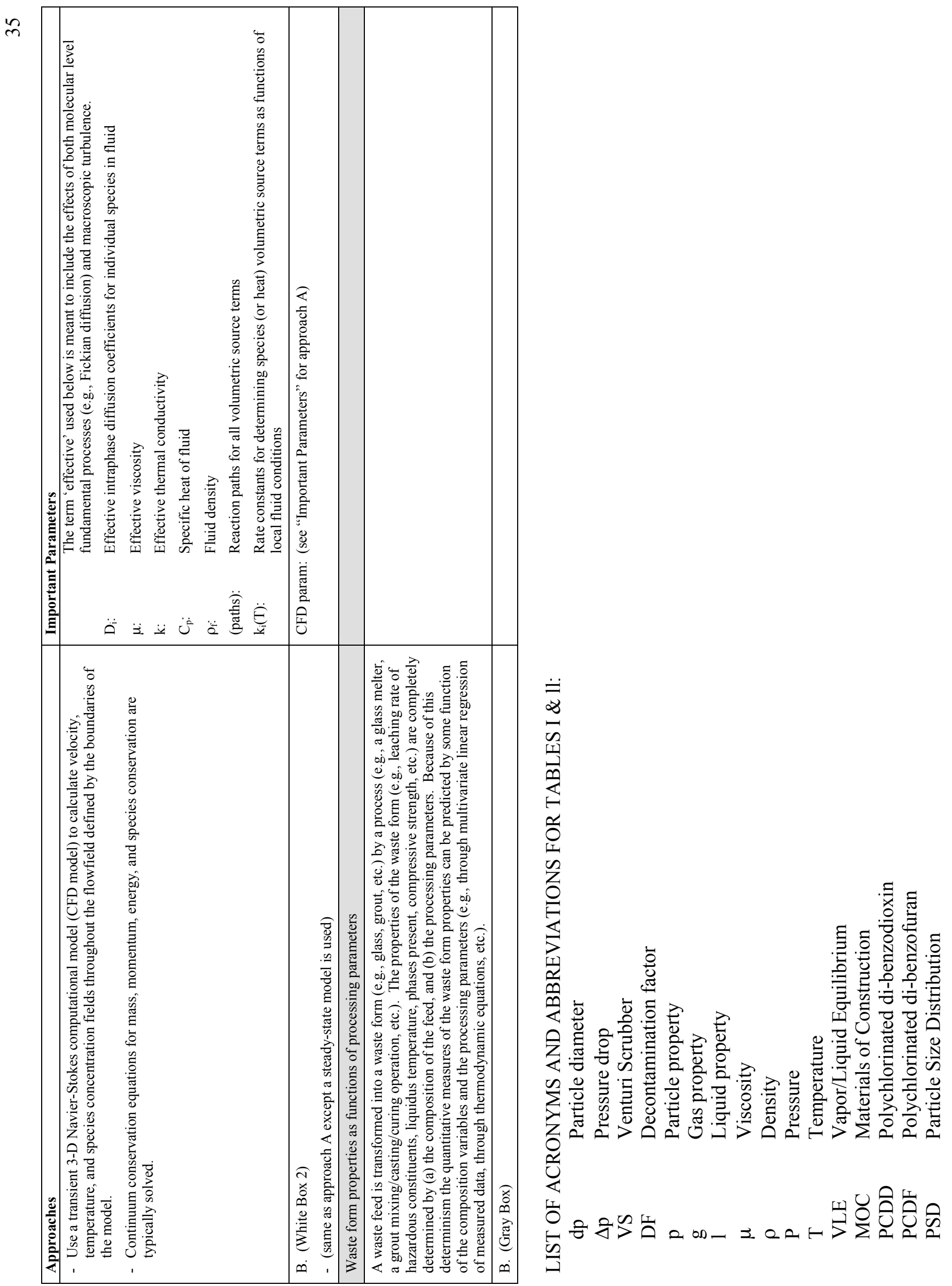
TABLE III: REPRESENTATIVE COMPOSITIONS OF DILUTE SBW FEED

\begin{tabular}{|c|c|c|c|c|c|}
\hline & & $\underline{\text { Units }}$ & $\underline{W M-181}$ & $\underline{W M-184}$ & $\underline{W M-186}$ \\
\hline \multicolumn{6}{|c|}{ Physical Characteristics } \\
\hline Volume & Vol. & US gallons & 275900 & 262600 & 281500 \\
\hline Specific Gravity & SpGr & $\mathrm{g} / \mathrm{ml}$ & 1.150518583 & 1.218753333 & 1.15832 \\
\hline Undissolved Solids & UDS & $\mathrm{g} / 1$ & 8.965714286 & $<$ visible & \#N/A \\
\hline \multicolumn{6}{|l|}{ Elements } \\
\hline Acid & $\mathrm{H}^{+}$ & Normal Acid & 1.643 & 1.673333333 & 1.363333333 \\
\hline Aluminum & $\mathrm{Al}$ & Molar & 0.211375 & 0.507033333 & 0.2637 \\
\hline Antimony & $\mathrm{Sb}$ & Molar & $3.28561 \mathrm{E}-08$ & $2.04119 \mathrm{E}-08$ & $9.19611 \mathrm{E}-10$ \\
\hline Arsenic & As & Molar & $<3.82 \mathrm{E}-5 \mathrm{M}$ & $7.62954 \mathrm{E}-10$ & $3.43731 \mathrm{E}-11$ \\
\hline Barium & $\mathrm{Ba}$ & Molar & $3.69058 \mathrm{E}-05$ & No Est. & No Est. \\
\hline Beryllium & $\mathrm{Be}$ & Molar & $8.43401 \mathrm{E}-12$ & $5.23964 \mathrm{E}-12$ & $2.3606 \mathrm{E}-13$ \\
\hline Boron & $\mathrm{B}$ & Molar & 0.016074344 & $<1.39 \mathrm{E}-02 \mathrm{M}$ & $<1.39 \mathrm{E}-02 \mathrm{M}$ \\
\hline Bromine & $\mathrm{Br}$ & Molar & $1.55204 \mathrm{E}-07$ & $9.64208 \mathrm{E}-08$ & $4.34401 \mathrm{E}-09$ \\
\hline Cadmium & $\mathrm{Cd}$ & Molar & 0.005014523 & $<4.7 \mathrm{E}-03 \mathrm{M}$ & $<4.70 \mathrm{E}-03 \mathrm{M}$ \\
\hline Calcium & $\mathrm{Ca}$ & Molar & 0.04457752 & 0.016033333 & 0.0498 \\
\hline Cerium & $\mathrm{Ce}$ & Molar & $9.13642 \mathrm{E}-06$ & $5.67601 \mathrm{E}-06$ & $2.55719 \mathrm{E}-07$ \\
\hline Chloride & $\mathrm{Cl}$ & Molar & 0.010143797 & 0.02753904 & 0.018014613 \\
\hline Chromium & $\mathrm{Cr}$ & Molar & 0.0027228 & $<4.0 \mathrm{E}-3 \mathrm{M}$ & 0.004133333 \\
\hline Dysprosium & Dy & Molar & $3.20037 \mathrm{E}-10$ & $1.98823 \mathrm{E}-10$ & $8.95753 \mathrm{E}-12$ \\
\hline Erbium & Er & Molar & $5.26188 \mathrm{E}-12$ & $3.26895 \mathrm{E}-12$ & $1.47275 \mathrm{E}-13$ \\
\hline Fluoride & $\mathrm{F}$ & Molar & 0.088557909 & 0.023159841 & 0.039827909 \\
\hline Gadolinium & $\mathrm{Gd}$ & Molar & $1.32289 \mathrm{E}-07$ & $8.21846 \mathrm{E}-08$ & 3.70263E-09 \\
\hline Gallium & $\mathrm{Ga}$ & Molar & $9.75402 \mathrm{E}-15$ & $6.05969 \mathrm{E}-15$ & $2.73005 \mathrm{E}-16$ \\
\hline Germanium & $\mathrm{Ge}$ & Molar & $4.51781 \mathrm{E}-09$ & $2.80669 \mathrm{E}-09$ & $1.26449 \mathrm{E}-10$ \\
\hline Holmium & Ho & Molar & $1.35831 \mathrm{E}-11$ & $8.4385 \mathrm{E}-12$ & $3.80177 \mathrm{E}-13$ \\
\hline Iodine & I & Molar & 0.000182836 & 0.000113587 & 5.1174E-06 \\
\hline Iron & $\mathrm{Fe}$ & Molar & 0.01168553 & 0.014566667 & 0.015633333 \\
\hline Lanthanum & $\mathrm{La}$ & Molar & 4.60799E-06 & $2.86271 \mathrm{E}-06$ & $1.28973 \mathrm{E}-07$ \\
\hline Lead & $\mathrm{Pb}$ & Molar & 0.000994553 & 0.000283102 & 0.000417849 \\
\hline Lithium & $\mathrm{Li}$ & Molar & $2.01724 \mathrm{E}-11$ & $1.25321 \mathrm{E}-11$ & $5.64604 \mathrm{E}-13$ \\
\hline Manganese & $\mathrm{Mn}$ & Molar & 0.012952771 & No Est. & No Est. \\
\hline Mercury & $\mathrm{Hg}$ & Molar & 0.000480369 & 0.000686309 & 0.00094222 \\
\hline Molybdenum & Mo & Molar & 0.000521159 & $1.1398 \mathrm{E}-05$ & $5.13513 \mathrm{E}-07$ \\
\hline Neodymium & $\mathrm{Nd}$ & Molar & $1.52541 \mathrm{E}-05$ & $9.47664 \mathrm{E}-06$ & $4.26948 \mathrm{E}-07$ \\
\hline Nickel & $\mathrm{Ni}$ & Molar & 0.001152972 & $<4.14 \mathrm{E}-3 \mathrm{M}$ & $<4.14 \mathrm{E}-03 \mathrm{M}$ \\
\hline Nitrate & NO3 & Molar & 3.388 & 3.99 & 2.945 \\
\hline Palladium & $\mathrm{Pd}$ & Molar & $1.80438 \mathrm{E}-06$ & $1.12097 \mathrm{E}-06$ & $5.05029 \mathrm{E}-08$ \\
\hline Phosphorus & $\mathrm{P}$ & Molar & 0.006134224 & 0.010499607 & 0.000494051 \\
\hline Plutonium & $\mathrm{Pu}$ & Molar & $1.9837 \mathrm{E}-06$ & $1.23238 \mathrm{E}-06$ & $5.55219 \mathrm{E}-08$ \\
\hline Potassium & K & Molar & 0.121087848 & 0.084658412 & 0.139136484 \\
\hline Praseodymium & $\operatorname{Pr}$ & Molar & $4.2586 \mathrm{E}-06$ & $2.64566 \mathrm{E}-06$ & $1.19194 \mathrm{E}-07$ \\
\hline Rhodium & $\mathrm{Rh}$ & Molar & $1.86601 \mathrm{E}-06$ & $1.15926 \mathrm{E}-06$ & $5.22277 \mathrm{E}-08$ \\
\hline Rubidium & $\mathrm{Rb}$ & Molar & $2.85521 \mathrm{E}-06$ & $1.7738 \mathrm{E}-06$ & $7.99145 \mathrm{E}-08$ \\
\hline Ruthenium & $\mathrm{Ru}$ & Molar & $8.70785 \mathrm{E}-06$ & $5.40976 \mathrm{E}-06$ & $2.43724 \mathrm{E}-07$ \\
\hline Samarium & $\mathrm{Sm}$ & Molar & $2.92665 \mathrm{E}-06$ & $1.81818 \mathrm{E}-06$ & $8.19141 \mathrm{E}-08$ \\
\hline Selenium & $\mathrm{Se}$ & Molar & $<1.25 \mathrm{E}-5 \mathrm{M}$ & $2.5495 \mathrm{E}-07$ & $1.14862 \mathrm{E}-08$ \\
\hline
\end{tabular}




\begin{tabular}{|c|c|c|c|c|c|}
\hline Silver & $\mathrm{Ag}$ & Molar & $3.38608 \mathrm{E}-05$ & $2.02753 \mathrm{E}-08$ & $9.13455 \mathrm{E}-10$ \\
\hline Sodium & $\mathrm{Na}$ & Molar & 0.859915477 & 1.284629464 & 0.840953825 \\
\hline Sulfate & $\mathrm{SO} 4$ & Molar & 0.03826369 & 0.027366667 & 0.029077958 \\
\hline Technetium & $\mathrm{Tc}$ & Molar & $3.01066 \mathrm{E}-06$ & $1.87037 \mathrm{E}-06$ & $8.42654 \mathrm{E}-08$ \\
\hline Tellurium & $\mathrm{Te}$ & Molar & $1.47353 \mathrm{E}-06$ & $9.15429 \mathrm{E}-07$ & 4.12425E-08 \\
\hline Terbium & $\mathrm{Tb}$ & Molar & $1.0824 \mathrm{E}-09$ & $6.72439 \mathrm{E}-10$ & $3.02952 \mathrm{E}-11$ \\
\hline Thulium & $\mathrm{Tm}$ & Molar & $2.60487 \mathrm{E}-15$ & $1.61828 \mathrm{E}-15$ & 7.29077E-17 \\
\hline Tin & $\mathrm{Sn}$ & Molar & $1.68497 \mathrm{E}-07$ & $1.04679 \mathrm{E}-07$ & 4.71608E-09 \\
\hline Uranium & $\mathrm{U}$ & Molar & 0.000318727 & 0.000180953 & 0.000382231 \\
\hline Ytterbium & $\mathrm{Yb}$ & Molar & 4.39256E-16 & $2.72888 \mathrm{E}-16$ & $1.22943 \mathrm{E}-17$ \\
\hline Yttrium & Y & Molar & 3.50974E-06 & $2.18043 \mathrm{E}-06$ & $9.82341 \mathrm{E}-08$ \\
\hline Zirconium & $\mathrm{Zr}$ & Molar & 0.006062829 & $<1.21 \mathrm{E}-02 \mathrm{M}$ & $<1.2 \mathrm{e}-2 \mathrm{M}$ \\
\hline \multicolumn{6}{|c|}{ Actinides and Daughters } \\
\hline Thallium & Tl-207 & $\mathrm{mCi} / 1$ & $1.60019 \mathrm{E}-08$ & $9.94117 \mathrm{E}-09$ & $4.47876 \mathrm{E}-10$ \\
\hline Thallium & Tl-208 & $\mathrm{mCi} / 1$ & $1.12013 \mathrm{E}-06$ & $6.95882 \mathrm{E}-07$ & $3.13513 \mathrm{E}-08$ \\
\hline Lead & $\mathrm{Pb}-209$ & $\mathrm{mCi} / 1$ & $8.40098 \mathrm{E}-11$ & $5.21911 \mathrm{E}-11$ & $2.35135 \mathrm{E}-12$ \\
\hline Lead & $\mathrm{Pb}-210$ & $\mathrm{mCi} / 1$ & $1.08013 \mathrm{E}-09$ & $6.71029 \mathrm{E}-10$ & $3.02317 \mathrm{E}-11$ \\
\hline Lead & $\mathrm{Pb}-211$ & $\mathrm{mCi} / 1$ & $1.60019 \mathrm{E}-08$ & $9.94117 \mathrm{E}-09$ & $4.47876 \mathrm{E}-10$ \\
\hline Lead & $\mathrm{Pb}-212$ & $\mathrm{mCi} / 1$ & $3.16037 \mathrm{E}-06$ & $1.96338 \mathrm{E}-06$ & $8.84556 \mathrm{E}-08$ \\
\hline Lead & $\mathrm{Pb}-214$ & $\mathrm{mCi} / 1$ & $3.4404 \mathrm{E}-09$ & $2.13735 \mathrm{E}-09$ & $9.62934 \mathrm{E}-11$ \\
\hline Bismuth & $\mathrm{Bi}-210 \mathrm{~m}$ & $\mathrm{mCi} / 1$ & $1.08013 \mathrm{E}-22$ & $6.71029 \mathrm{E}-23$ & $3.02317 \mathrm{E}-24$ \\
\hline Bismuth & $\mathrm{Bi}-210$ & $\mathrm{mCi} / 1$ & $1.08013 \mathrm{E}-09$ & $6.71029 \mathrm{E}-10$ & $3.02317 \mathrm{E}-11$ \\
\hline Bismuth & $\mathrm{Bi}-211$ & $\mathrm{mCi} / 1$ & $1.60019 \mathrm{E}-08$ & $9.94117 \mathrm{E}-09$ & $4.47876 \mathrm{E}-10$ \\
\hline Bismuth & $\mathrm{Bi}-212$ & $\mathrm{mCi} / 1$ & $3.16037 \mathrm{E}-06$ & $1.96338 \mathrm{E}-06$ & $8.84556 \mathrm{E}-08$ \\
\hline Bismuth & $\mathrm{Bi}-213$ & $\mathrm{mCi} / 1$ & $8.40098 \mathrm{E}-11$ & $5.21911 \mathrm{E}-11$ & $2.35135 \mathrm{E}-12$ \\
\hline Bismuth & $\mathrm{Bi}-214$ & $\mathrm{mCi} / 1$ & $3.4404 \mathrm{E}-09$ & $2.13735 \mathrm{E}-09$ & $9.62934 \mathrm{E}-11$ \\
\hline Polonium & Po-210 & $\mathrm{mCi} / 1$ & $1.04012 \mathrm{E}-09$ & $6.46176 \mathrm{E}-10$ & $2.9112 \mathrm{E}-11$ \\
\hline Polonium & Рo-213 & $\mathrm{mCi} / 1$ & $8.40098 \mathrm{E}-11$ & $5.21911 \mathrm{E}-11$ & $2.35135 \mathrm{E}-12$ \\
\hline Polonium & Po-214 & $\mathrm{mCi} / 1$ & $3.4404 \mathrm{E}-09$ & $2.13735 \mathrm{E}-09$ & $9.62934 \mathrm{E}-11$ \\
\hline Polonium & Po-215 & $\mathrm{mCi} / 1$ & $1.60019 \mathrm{E}-08$ & $9.94117 \mathrm{E}-09$ & $4.47876 \mathrm{E}-10$ \\
\hline Polonium & Po-216 & $\mathrm{mCi} / 1$ & $3.16037 \mathrm{E}-06$ & $1.96338 \mathrm{E}-06$ & $8.84556 \mathrm{E}-08$ \\
\hline Polonium & Po-218 & $\mathrm{mCi} / 1$ & $3.4404 \mathrm{E}-09$ & $2.13735 \mathrm{E}-09$ & $9.62934 \mathrm{E}-11$ \\
\hline Astatine & At-217 & $\mathrm{mCi} / 1$ & $8.40098 \mathrm{E}-11$ & $5.21911 \mathrm{E}-11$ & $2.35135 \mathrm{E}-12$ \\
\hline Radon & Rn-219 & $\mathrm{mCi} / 1$ & $1.60019 \mathrm{E}-08$ & $9.94117 \mathrm{E}-09$ & $4.47876 \mathrm{E}-10$ \\
\hline Radon & Rn-220 & $\mathrm{mCi} / 1$ & $3.16037 \mathrm{E}-06$ & $1.96338 \mathrm{E}-06$ & $8.84556 \mathrm{E}-08$ \\
\hline Radon & Rn-222 & $\mathrm{mCi} / 1$ & $3.4404 \mathrm{E}-09$ & $2.13735 \mathrm{E}-09$ & $9.62934 \mathrm{E}-11$ \\
\hline Francium & Fr-221 & $\mathrm{mCi} / 1$ & $8.40098 \mathrm{E}-11$ & $5.21911 \mathrm{E}-11$ & $2.35135 \mathrm{E}-12$ \\
\hline Francium & Fr-223 & $\mathrm{mCi} / 1$ & $2.24026 \mathrm{E}-10$ & $1.39176 \mathrm{E}-10$ & $6.27027 \mathrm{E}-12$ \\
\hline Radium & Ra-223 & $\mathrm{mCi} / 1$ & $1.60019 \mathrm{E}-08$ & $9.94117 \mathrm{E}-09$ & $4.47876 \mathrm{E}-10$ \\
\hline Radium & Ra-224 & $\mathrm{mCi} / 1$ & $3.16037 \mathrm{E}-06$ & $1.96338 \mathrm{E}-06$ & $8.84556 \mathrm{E}-08$ \\
\hline Radium & Ra-225 & $\mathrm{mCi} / 1$ & $8.40098 \mathrm{E}-11$ & $5.21911 \mathrm{E}-11$ & $2.35135 \mathrm{E}-12$ \\
\hline Radium & Ra-226 & $\mathrm{mCi} / 1$ & 3.4404E-09 & $2.13735 \mathrm{E}-09$ & $9.62934 \mathrm{E}-11$ \\
\hline Radium & $\mathrm{Ra}-228$ & $\mathrm{mCi} / 1$ & $2.28027 \mathrm{E}-13$ & $1.41662 \mathrm{E}-13$ & $6.38224 \mathrm{E}-15$ \\
\hline Actinium & Ac-225 & $\mathrm{mCi} / 1$ & $8.40098 \mathrm{E}-11$ & $5.21911 \mathrm{E}-11$ & $2.35135 \mathrm{E}-12$ \\
\hline Actinium & Ac-227 & $\mathrm{mCi} / 1$ & 1.60019E-08 & $9.94117 \mathrm{E}-09$ & $4.47876 \mathrm{E}-10$ \\
\hline Actinium & Ac-228 & $\mathrm{mCi} / 1$ & $2.28027 \mathrm{E}-13$ & $1.41662 \mathrm{E}-13$ & $6.38224 \mathrm{E}-15$ \\
\hline Thorium & Th-227 & $\mathrm{mCi} / 1$ & $1.60019 \mathrm{E}-08$ & $9.94117 \mathrm{E}-09$ & $4.47876 \mathrm{E}-10$ \\
\hline Thorium & Th-228 & $\mathrm{mCi} / 1$ & $3.16037 \mathrm{E}-06$ & $1.96338 \mathrm{E}-06$ & $8.84556 \mathrm{E}-08$ \\
\hline Thorium & Th-229 & $\mathrm{mCi} / 1$ & $8.40098 \mathrm{E}-11$ & $5.21911 \mathrm{E}-11$ & $2.35135 \mathrm{E}-12$ \\
\hline Thorium & Th-230 & $\mathrm{mCi} / 1$ & $3.96046 \mathrm{E}-07$ & $2.46044 \mathrm{E}-07$ & $1.10849 \mathrm{E}-08$ \\
\hline Thorium & Th-231 & $\mathrm{mCi} / 1$ & $1.04012 \mathrm{E}-05$ & $6.46176 \mathrm{E}-06$ & $2.9112 \mathrm{E}-07$ \\
\hline
\end{tabular}




\begin{tabular}{|c|c|c|c|c|c|}
\hline Thorium & Th-232 & $\mathrm{mCi} / 1$ & $3.48041 \mathrm{E}-13$ & $2.1622 \mathrm{E}-13$ & $9.74131 \mathrm{E}-15$ \\
\hline Thorium & Th-234 & $\mathrm{mCi} / 1$ & $1.04012 \mathrm{E}-05$ & $6.46176 \mathrm{E}-06$ & $2.9112 \mathrm{E}-07$ \\
\hline Protactinium & $\mathrm{Pa}-231$ & $\mathrm{mCi} / 1$ & 4.40051E-08 & $2.73382 \mathrm{E}-08$ & $1.23166 \mathrm{E}-09$ \\
\hline Protactinium & $\mathrm{Pa}-233$ & $\mathrm{mCi} / 1$ & 0.001440168 & 0.000894705 & 4.03089E-05 \\
\hline Protactinium & $\mathrm{Pa}-234 \mathrm{~m}$ & $\mathrm{mCi} / 1$ & $1.04012 \mathrm{E}-05$ & $6.46176 \mathrm{E}-06$ & $2.9112 \mathrm{E}-07$ \\
\hline Protactinium & $\mathrm{Pa}-234$ & $\mathrm{mCi} / 1$ & $1.32015 \mathrm{E}-08$ & 8.20147E-09 & $3.69498 \mathrm{E}-10$ \\
\hline Uranium & $\mathrm{U}-232$ & $\mathrm{mCi} / 1$ & $9.60112 \mathrm{E}-07$ & $5.9647 \mathrm{E}-07$ & $2.68726 \mathrm{E}-08$ \\
\hline Uranium & U-233 & $\mathrm{mCi} / 1$ & $1.60019 \mathrm{E}-08$ & $9.94117 \mathrm{E}-09$ & $4.47876 \mathrm{E}-10$ \\
\hline Uranium & U-234 & $\mathrm{mCi} / 1$ & 0.000400047 & 0.001074804 & 2.11479E-09 \\
\hline Uranium & U-235 & $\mathrm{mCi} / 1$ & $1.04012 \mathrm{E}-05$ & $2.81214 \mathrm{E}-05$ & $1.53625 \mathrm{E}-07$ \\
\hline Uranium & U-236 & $\mathrm{mCi} / 1$ & $1.64019 \mathrm{E}-05$ & $5.51244 \mathrm{E}-05$ & 1.48493E-08 \\
\hline Uranium & $\mathrm{U}-237$ & $\mathrm{mCi} / 1$ & $3.76044 \mathrm{E}-06$ & $2.33618 \mathrm{E}-06$ & $1.05251 \mathrm{E}-07$ \\
\hline Uranium & $\mathrm{U}-238$ & $\mathrm{mCi} / 1$ & $1.04012 \mathrm{E}-05$ & $9.75972 \mathrm{E}-06$ & $1.95203 \mathrm{E}-06$ \\
\hline Uranium & $\mathrm{U}-240$ & $\mathrm{mCi} / 1$ & $3.36039 \mathrm{E}-13$ & $2.08765 \mathrm{E}-13$ & $9.4054 \mathrm{E}-15$ \\
\hline Neptunium & Np-237 & $\mathrm{mCi} / 1$ & 0.000832851 & 0.000894705 & 4.03089E-05 \\
\hline Neptunium & Np-238 & $\mathrm{mCi} / \mathrm{l}$ & $3.84045 \mathrm{E}-08$ & $2.38588 \mathrm{E}-08$ & $1.0749 \mathrm{E}-09$ \\
\hline Neptunium & Np-239 & $\mathrm{mCi} / 1$ & $1.08013 \mathrm{E}-05$ & $6.71029 \mathrm{E}-06$ & $3.02317 \mathrm{E}-07$ \\
\hline Neptunium & $\mathrm{Np}-240 \mathrm{~m}$ & $\mathrm{mCi} / 1$ & $3.36039 \mathrm{E}-13$ & $2.08765 \mathrm{E}-13$ & $9.4054 \mathrm{E}-15$ \\
\hline Plutonium & $\mathrm{Pu}-236$ & $\mathrm{mCi} / 1$ & $3.16037 \mathrm{E}-06$ & $1.96338 \mathrm{E}-06$ & $8.84556 \mathrm{E}-08$ \\
\hline Plutonium & $\mathrm{Pu}-238$ & $\mathrm{mCi} / 1$ & 0.593343756 & 0.336306306 & 0.005374516 \\
\hline Plutonium & $\mathrm{Pu}-239$ & $\mathrm{mCi} / 1$ & 0.015315081 & 0.062972973 & 0.000750193 \\
\hline Plutonium & $\mathrm{Pu}-240$ & $\mathrm{mCi} / 1$ & 0.003698017 & 0.00323088 & 0.00014556 \\
\hline Plutonium & $\mathrm{Pu}-241$ & $\mathrm{mCi} / 1$ & 0.442087294 & 0.094441117 & 0.004254825 \\
\hline Plutonium & $\mathrm{Pu}-242$ & $\mathrm{mCi} / 1$ & $8.73507 \mathrm{E}-06$ & $2.43559 \mathrm{E}-06$ & $1.0973 \mathrm{E}-07$ \\
\hline Plutonium & $\mathrm{Pu}-244$ & $\mathrm{mCi} / 1$ & $3.36039 \mathrm{E}-13$ & $2.08765 \mathrm{E}-13$ & $9.4054 \mathrm{E}-15$ \\
\hline Americium & Am-241 & $\mathrm{mCi} / 1$ & 0.036199679 & 0.075945946 & 0.000671814 \\
\hline Americium & $\mathrm{Am}-242 \mathrm{~m}$ & $\mathrm{mCi} / 1$ & $7.60089 \mathrm{E}-06$ & $4.72206 \mathrm{E}-06$ & $2.12741 \mathrm{E}-07$ \\
\hline Americium & Am-242 & $\mathrm{mCi} / 1$ & $7.60089 \mathrm{E}-06$ & $4.72206 \mathrm{E}-06$ & $2.12741 \mathrm{E}-07$ \\
\hline Americium & Am-243 & $\mathrm{mCi} / 1$ & $1.08013 \mathrm{E}-05$ & $6.71029 \mathrm{E}-06$ & $3.02317 \mathrm{E}-07$ \\
\hline Curium & $\mathrm{Cm}-242$ & $\mathrm{mCi} / 1$ & $1.08013 \mathrm{E}-05$ & $6.71029 \mathrm{E}-06$ & 3.02317E-07 \\
\hline Curium & $\mathrm{Cm}-243$ & $\mathrm{mCi} / 1$ & $1.52018 \mathrm{E}-05$ & $9.44411 \mathrm{E}-06$ & 4.25483E-07 \\
\hline Curium & $\mathrm{Cm}-244$ & $\mathrm{mCi} / 1$ & 0.001000117 & 0.000621323 & 2.79923E-05 \\
\hline Curium & $\mathrm{Cm}-245$ & $\mathrm{mCi} / 1$ & $1.48017 \mathrm{E}-07$ & $9.19558 \mathrm{E}-08$ & 4.14286E-09 \\
\hline Curium & $\mathrm{Cm}-246$ & $\mathrm{mCi} / 1$ & $9.60112 \mathrm{E}-09$ & 5.9647E-09 & $2.68726 \mathrm{E}-10$ \\
\hline Curium & $\mathrm{Cm}-247$ & $\mathrm{mCi} / 1$ & $1.08013 \mathrm{E}-14$ & $6.71029 \mathrm{E}-15$ & 3.02317E-16 \\
\hline Curium & $\mathrm{Cm}-248$ & $\mathrm{mCi} / 1$ & $1.16014 \mathrm{E}-14$ & $7.20735 \mathrm{E}-15$ & $3.2471 \mathrm{E}-16$ \\
\hline Californium & Cf-249 & $\mathrm{mCi} / 1$ & $8.80103 \mathrm{E}-15$ & $5.46764 \mathrm{E}-15$ & $2.46332 \mathrm{E}-16$ \\
\hline Californium & Cf-250 & $\mathrm{mCi} / 1$ & $8.40098 \mathrm{E}-15$ & $5.21911 \mathrm{E}-15$ & $2.35135 \mathrm{E}-16$ \\
\hline Californium & Cf-251 & $\mathrm{mCi} / 1$ & $1.36016 \mathrm{E}-16$ & $8.44999 \mathrm{E}-17$ & $3.80695 \mathrm{E}-18$ \\
\hline \multicolumn{6}{|c|}{ Fission Products } \\
\hline Tritium & $\mathrm{H}-3$ & $\mathrm{mCi} / 1$ & 0.028656685 & 0.004224997 & 0.000190347 \\
\hline Beryllium & $\mathrm{Be}-10$ & $\mathrm{mCi} / 1$ & $1.48017 \mathrm{E}-09$ & $9.19558 \mathrm{E}-10$ & $4.14286 \mathrm{E}-11$ \\
\hline Carbon & $\mathrm{C}-14$ & $\mathrm{mCi} / 1$ & $6.0007 \mathrm{E}-08$ & $3.72794 \mathrm{E}-08$ & 1.67954E-09 \\
\hline Selenium & Se-79 & $\mathrm{mCi} / 1$ & 0.000216025 & 0.000134206 & $6.04633 \mathrm{E}-06$ \\
\hline Rubidium & $\mathrm{Rb}-87$ & $\mathrm{mCi} / 1$ & $1.44017 \mathrm{E}-08$ & $8.94705 \mathrm{E}-09$ & $4.03089 \mathrm{E}-10$ \\
\hline Strontium & Sr-90 & $\mathrm{mCi} / 1$ & 25.07736436 & 13.44260157 & 20.38024919 \\
\hline Yttrium & Y-90 & $\mathrm{mCi} / 1$ & 27.60321652 & 17.14851862 & 0.772586656 \\
\hline Zirconium & Zr-93 & $\mathrm{mCi} / 1$ & 0.001080126 & 0.000671029 & $3.02317 \mathrm{E}-05$ \\
\hline Niobium & $\mathrm{Nb}-93 \mathrm{~m}$ & $\mathrm{mCi} / 1$ & 0.000800093 & 0.000497059 & $2.23938 \mathrm{E}-05$ \\
\hline Niobium & $\mathrm{Nb}-94$ & $\mathrm{mCi} / 1$ & 0.000560065 & 0.000347941 & $1.56757 \mathrm{E}-05$ \\
\hline Zirconium & $\mathrm{Zr}-95$ & $\mathrm{mCi} / 1$ & 0.02972973 & & \\
\hline
\end{tabular}




\begin{tabular}{|c|c|c|c|c|c|}
\hline Technetium & Tc-98 & $\mathrm{mCi} / 1$ & $1.28015 \mathrm{E}-09$ & 7.95294E-10 & $3.58301 \mathrm{E}-11$ \\
\hline Technetium & Tc-99 & $\mathrm{mCi} / 1$ & 0.004800559 & 0.002982351 & 0.000134363 \\
\hline Ruthenium & Ru-106 & $\mathrm{mCi} / 1$ & 0.001871889 & 0.00323088 & 0.00014556 \\
\hline Rhodium & Rh-102 & $\mathrm{mCi} / 1$ & $1.00012 \mathrm{E}-06$ & $6.21323 \mathrm{E}-07$ & 2.79923E-08 \\
\hline Rhodium & Rh-106 & $\mathrm{mCi} / 1$ & 0.005200606 & 0.00323088 & 0.00014556 \\
\hline Palladium & Pd-107 & $\mathrm{mCi} / 1$ & 8.00093E-06 & 4.97059E-06 & $2.23938 \mathrm{E}-07$ \\
\hline Cadmium & Cd-113m & $\mathrm{mCi} / 1$ & 0.001960228 & 0.001217793 & $5.48648 \mathrm{E}-05$ \\
\hline Indium & In-115 & $\mathrm{mCi} / 1$ & 4.80056E-14 & $2.98235 \mathrm{E}-14$ & $1.34363 \mathrm{E}-15$ \\
\hline Tin & Sn-121m & $\mathrm{mCi} / 1$ & $3.48041 \mathrm{E}-05$ & $2.1622 \mathrm{E}-05$ & $9.74131 \mathrm{E}-07$ \\
\hline Tin & Sn-126 & $\mathrm{mCi} / 1$ & 0.000204024 & 0.00012675 & 5.71042E-06 \\
\hline Antimony & Sb-125 & $\mathrm{mCi} / 1$ & 0.06694425 & 0.009444112 & 0.000425483 \\
\hline Antimony & $\mathrm{Sb}-126 \mathrm{~m}$ & $\mathrm{mCi} / 1$ & 0.000204024 & 0.00012675 & 5.71042E-06 \\
\hline Antimony & $\mathrm{Sb}-126$ & $\mathrm{mCi} / 1$ & $2.84033 \mathrm{E}-05$ & $1.76456 \mathrm{E}-05$ & $7.9498 \mathrm{E}-07$ \\
\hline Tellurium & Te-123 & $\mathrm{mCi} / 1$ & $1.88022 \mathrm{E}-16$ & $1.16809 \mathrm{E}-16$ & $5.26255 \mathrm{E}-18$ \\
\hline Tellurium & Te- $125 \mathrm{~m}$ & $\mathrm{mCi} / 1$ & 0.003720434 & 0.002311322 & 0.000104131 \\
\hline Iodine & I-129 & $\mathrm{mCi} / 1$ & $<$ check logs & $6.64865 \mathrm{E}-05$ & 7.38996E-07 \\
\hline Cesium & Cs-134 & $\mathrm{mCi} / 1$ & 0.158675081 & 0.008917964 & 0.630229045 \\
\hline Cesium & Cs-135 & $\mathrm{mCi} / 1$ & 0.000440051 & 0.000273382 & $1.23166 \mathrm{E}-05$ \\
\hline Cesium & Cs-137 & $\mathrm{mCi} / 1$ & 27.05515266 & 16.80803354 & 0.757246892 \\
\hline Barium & Ba-137m & $\mathrm{mCi} / 1$ & 25.60298344 & 15.90587234 & 0.716602116 \\
\hline Lanthanum & La-138 & $\mathrm{mCi} / 1$ & $9.60112 \mathrm{E}-14$ & $5.9647 \mathrm{E}-14$ & $2.68726 \mathrm{E}-15$ \\
\hline Cerium & Ce-142 & $\mathrm{mCi} / 1$ & 1.48017E-08 & $9.19558 \mathrm{E}-09$ & 4.14286E-10 \\
\hline Cerium & Ce-144 & $\mathrm{mCi} / 1$ & 0.000532123 & 0.004473527 & 0.000201544 \\
\hline Praseodymium & Pr-144 & $\mathrm{mCi} / 1$ & $8.40098 \mathrm{E}-05$ & $5.21911 \mathrm{E}-05$ & 2.35135E-06 \\
\hline Neodymium & Nd-144 & $\mathrm{mCi} / 1$ & $8.00093 \mathrm{E}-13$ & $4.97059 \mathrm{E}-13$ & $2.23938 \mathrm{E}-14$ \\
\hline Promethium & Pm-146 & $\mathrm{mCi} / 1$ & $3.92046 \mathrm{E}-05$ & $2.43559 \mathrm{E}-05$ & $1.0973 \mathrm{E}-06$ \\
\hline Promethium & Pm-147 & $\mathrm{mCi} / 1$ & 0.212024707 & 0.131720505 & 0.005934361 \\
\hline Samarium & Sm-146 & $\mathrm{mCi} / 1$ & $1.36016 \mathrm{E}-10$ & $8.44999 \mathrm{E}-11$ & 3.80695E-12 \\
\hline Samarium & Sm-147 & $\mathrm{mCi} / 1$ & 3.64042E-09 & $2.26162 \mathrm{E}-09$ & $1.01892 \mathrm{E}-10$ \\
\hline Samarium & Sm-148 & $\mathrm{mCi} / 1$ & $1.88022 \mathrm{E}-14$ & $1.16809 \mathrm{E}-14$ & $5.26255 \mathrm{E}-16$ \\
\hline Samarium & Sm-149 & $\mathrm{mCi} / 1$ & $1.6802 \mathrm{E}-15$ & $1.04382 \mathrm{E}-15$ & $4.7027 \mathrm{E}-17$ \\
\hline Samarium & Sm-151 & $\mathrm{mCi} / 1$ & 0.172020045 & 0.10686758 & 0.00481467 \\
\hline Europium & Eu-150 & $\mathrm{mCi} / 1$ & 7.60089E-09 & 4.72206E-09 & $2.12741 \mathrm{E}-10$ \\
\hline Europium & Eu-152 & $\mathrm{mCi} / 1$ & 0.001480172 & 0.000919558 & 4.14286E-05 \\
\hline Europium & Eu-154 & $\mathrm{mCi} / 1$ & 0.266571307 & 0.032576432 & 0.108363634 \\
\hline Europium & Eu-155 & $\mathrm{mCi} / 1$ & 0.087507027 & 0.082014654 & 0.043578989 \\
\hline Gadolinium & Gd-152 & $\mathrm{mCi} / 1$ & $6.80079 \mathrm{E}-16$ & $4.225 \mathrm{E}-16$ & $1.90347 \mathrm{E}-17$ \\
\hline Holmium & Ho-166m & $\mathrm{mCi} / 1$ & $2.28027 \mathrm{E}-08$ & $1.41662 \mathrm{E}-08$ & $6.38224 \mathrm{E}-10$ \\
\hline Thulium & Tm-171 & $\mathrm{mCi} / 1$ & $8.80103 \mathrm{E}-13$ & $5.46764 \mathrm{E}-13$ & $2.46332 \mathrm{E}-14$ \\
\hline \multicolumn{6}{|c|}{ Activation Products } \\
\hline Cobalt & Co- 60 & $\mathrm{mCi} / 1$ & 0.099614336 & 0.025282202 & 0.767567568 \\
\hline Nickel & $\mathrm{Ni}-63$ & $\mathrm{mCi} / 1$ & 0.058870944 & 0.00994117 & 0.000447876 \\
\hline
\end{tabular}


TABLE IV: REPRESENTATIVE COMPOSITIONS IN SBW TANK

\begin{tabular}{|c|c|c|c|c|c|}
\hline \multicolumn{2}{|c|}{ Radionuclides decayed to $7 / 1 / 99$} & WM-180 & WM-188 & WM-189 & Total or Average \\
\hline \multicolumn{2}{|c|}{ Earliest date composition valid } & Jun-00 & Jun-05 & Apr-05 & \\
\hline & Volume, gallons & 284,550 & 288,000 & 296,000 & 868,550 \\
\hline & Density, g/cm3 & 1.26 & 1.28 & 1.35 & 1.30 \\
\hline & UDS, g/liter & 0.62 & 2.56 & 1.94 & 1.72 \\
\hline & TOC, g/liter & & 0.60 & 10.40 & 3.74 \\
\hline \multirow{2}{*}{\multicolumn{2}{|c|}{$\mathrm{pH}$}} & -0.05 & -0.41 & -0.37 & -0.28 \\
\hline & & Moles/liter & Moles/liter & Moles/liter & Moles/liter \\
\hline $\mathrm{H}+$ & Acid & $1.13 \mathrm{E}+00$ & $2.56 \mathrm{E}+00$ & $2.35 \mathrm{E}+00$ & $2.02 \mathrm{E}+00$ \\
\hline $\mathrm{Ac}+3$ & Actinium & $9.53 \mathrm{E}-16$ & $1.93 \mathrm{E}-15$ & $2.40 \mathrm{E}-15$ & $1.77 \mathrm{E}-15$ \\
\hline $\mathrm{Al}+3$ & Aluminum & $5.82 \mathrm{E}-01$ & $5.40 \mathrm{E}-01$ & 9.99E-01 & 7.10E-01 \\
\hline $\mathrm{Am}+4$ & Americium & 7.52E-08 & $1.34 \mathrm{E}-07$ & $1.33 \mathrm{E}-07$ & $1.14 \mathrm{E}-07$ \\
\hline $\mathrm{Sb}+5$ & Antimony & 3.19E-08 & $1.20 \mathrm{E}-06$ & $2.50 \mathrm{E}-06$ & $1.26 \mathrm{E}-06$ \\
\hline $\mathrm{As}+5$ & Arsenic & 4.67E-05 & $1.31 \mathrm{E}-04$ & $2.30 \mathrm{E}-05$ & $6.67 \mathrm{E}-05$ \\
\hline At & Astatine & $2.36 \mathrm{E}-28$ & $4.76 \mathrm{E}-28$ & $5.93 \mathrm{E}-28$ & 4.37E-28 \\
\hline $\mathrm{Ba}+2$ & Barium & 5.04E-05 & $7.68 \mathrm{E}-05$ & $6.53 \mathrm{E}-05$ & $6.43 \mathrm{E}-05$ \\
\hline $\mathrm{Be}+2$ & Beryllium & 4.76E-09 & $3.34 \mathrm{E}-07$ & $1.26 \mathrm{E}-06$ & $5.41 \mathrm{E}-07$ \\
\hline $\mathrm{Bi}+5$ & Bismuth & $4.53 \mathrm{E}-18$ & $1.04 \mathrm{E}-17$ & $1.10 \mathrm{E}-17$ & 8.67E-18 \\
\hline$B+3$ & Boron & $1.01 \mathrm{E}-02$ & 2.64E-02 & $1.91 \mathrm{E}-02$ & $1.86 \mathrm{E}-02$ \\
\hline Br-1 & Bromine & $1.52 \mathrm{E}-07$ & $9.57 \mathrm{E}-05$ & 4.26E-07 & 3.19E-05 \\
\hline $\mathrm{Cd}+2$ & Cadmium & 7.64E-04 & 8.59E-03 & $7.23 \mathrm{E}-03$ & $5.56 \mathrm{E}-03$ \\
\hline $\mathrm{Ca}+2$ & Calcium & $3.35 \mathrm{E}-02$ & 8.98E-02 & 7.13E-02 & $6.50 \mathrm{E}-02$ \\
\hline $\mathrm{Cf}+3$ & Californium & $9.08 \mathrm{E}-21$ & $1.83 \mathrm{E}-20$ & $2.28 \mathrm{E}-20$ & $1.68 \mathrm{E}-20$ \\
\hline $\mathrm{Ce}+4$ & Cerium & 8.92E-06 & $2.06 \mathrm{E}-05$ & 2.09E-05 & $1.69 \mathrm{E}-05$ \\
\hline $\mathrm{Cs}+1$ & Cesium & 9.49E-06 & $2.18 \mathrm{E}-05$ & $2.30 \mathrm{E}-05$ & $1.82 \mathrm{E}-05$ \\
\hline Cl-1 & Chloride & $3.07 \mathrm{E}-02$ & $2.70 \mathrm{E}-02$ & $3.13 \mathrm{E}-02$ & $2.97 \mathrm{E}-02$ \\
\hline $\mathrm{Cr}+3$ & Chromium & $3.25 \mathrm{E}-03$ & $1.55 \mathrm{E}-02$ & $6.41 \mathrm{E}-03$ & $8.40 \mathrm{E}-03$ \\
\hline $\mathrm{Co}+2$ & Cobalt & & 4.92E-06 & 7.74E-06 & 4.27E-06 \\
\hline $\mathrm{Cu}+2$ & Copper & & $6.65 \mathrm{E}-06$ & $9.37 \mathrm{E}-05$ & $3.41 \mathrm{E}-05$ \\
\hline $\mathrm{Cm}+3$ & Curium & 4.77E-12 & $9.65 \mathrm{E}-12$ & $1.20 \mathrm{E}-11$ & $8.85 \mathrm{E}-12$ \\
\hline Dy+3 & Dysprosium & $3.14 \mathrm{E}-10$ & $7.26 \mathrm{E}-10$ & 7.64E-10 & $6.04 \mathrm{E}-10$ \\
\hline $\mathrm{Er}+3$ & Erbium & $5.16 \mathrm{E}-12$ & $1.19 \mathrm{E}-11$ & $1.26 \mathrm{E}-11$ & $9.93 \mathrm{E}-12$ \\
\hline $\mathrm{Eu}+3$ & Europium & $2.57 \mathrm{E}-07$ & 5.91E-07 & $6.22 \mathrm{E}-07$ & 4.92E-07 \\
\hline F-1 & Fluoride & 4.13E-02 & $9.41 \mathrm{E}-02$ & $1.11 \mathrm{E}-01$ & 8.26E-02 \\
\hline $\mathrm{Fr}+1$ & Francium & $2.76 \mathrm{E}-23$ & $5.57 \mathrm{E}-23$ & $6.93 \mathrm{E}-23$ & $5.11 \mathrm{E}-23$ \\
\hline $\mathrm{Gd}+3$ & Gadolinium & $3.29 \mathrm{E}-07$ & $3.20 \mathrm{E}-06$ & $3.11 \mathrm{E}-05$ & $1.18 \mathrm{E}-05$ \\
\hline $\mathrm{Ga}+3$ & Gallium & $9.56 \mathrm{E}-15$ & $2.21 \mathrm{E}-14$ & $2.33 \mathrm{E}-14$ & $1.84 \mathrm{E}-14$ \\
\hline $\mathrm{Ge}+4$ & Germanium & 4.43E-09 & 2.24E-07 & $1.09 \mathrm{E}-08$ & 7.95E-08 \\
\hline $\mathrm{Ho}+3$ & Holmium & $1.33 \mathrm{E}-11$ & $3.08 \mathrm{E}-11$ & $3.24 \mathrm{E}-11$ & $2.56 \mathrm{E}-11$ \\
\hline $\mathrm{In}+3$ & Indium & $6.70 \mathrm{E}-07$ & $1.35 \mathrm{E}-06$ & $1.69 \mathrm{E}-06$ & $1.24 \mathrm{E}-06$ \\
\hline $\mathrm{I}-1$ & Iodine & $1.18 \mathrm{E}-03$ & 4.26E-04 & 4.36E-04 & $6.76 \mathrm{E}-04$ \\
\hline $\mathrm{Fe}+3$ & Iron & $1.73 \mathrm{E}-02$ & $2.72 \mathrm{E}-02$ & $2.60 \mathrm{E}-02$ & $2.36 \mathrm{E}-02$ \\
\hline $\mathrm{La}+3$ & Lanthanum & 4.52E-06 & $1.04 \mathrm{E}-05$ & $1.10 \mathrm{E}-05$ & 8.69E-06 \\
\hline $\mathrm{Pb}+2$ & Lead & $1.22 \mathrm{E}-03$ & $1.13 \mathrm{E}-03$ & $9.66 \mathrm{E}-04$ & $1.10 \mathrm{E}-03$ \\
\hline $\mathrm{Li}+1$ & Lithium & $1.91 \mathrm{E}-07$ & $1.23 \mathrm{E}-06$ & $1.29 \mathrm{E}-05$ & 4.86E-06 \\
\hline $\mathrm{Mg}+2$ & Magnesium & & & $3.43 \mathrm{E}-04$ & $1.17 \mathrm{E}-04$ \\
\hline $\mathrm{Mn}+4$ & Manganese & & $2.24 \mathrm{E}-02$ & $1.97 \mathrm{E}-02$ & $1.41 \mathrm{E}-02$ \\
\hline
\end{tabular}




\begin{tabular}{|c|c|c|c|c|c|}
\hline $\mathrm{Hg}+2$ & Mercury & $9.60 \mathrm{E}-04$ & $2.00 \mathrm{E}-03$ & $2.48 \mathrm{E}-03$ & $1.82 \mathrm{E}-03$ \\
\hline $\mathrm{Mo}+6$ & Molybdenum & $1.80 \mathrm{E}-05$ & $3.52 \mathrm{E}-04$ & $3.16 \mathrm{E}-04$ & 2.30E-04 \\
\hline $\mathrm{Nd}+3$ & Neodymium & $1.49 \mathrm{E}-05$ & $3.46 \mathrm{E}-05$ & 3.64E-05 & $2.88 \mathrm{E}-05$ \\
\hline $\mathrm{Np}+4$ & Neptunium & $1.64 \mathrm{E}-05$ & $9.87 \mathrm{E}-06$ & $1.72 \mathrm{E}-05$ & $1.45 \mathrm{E}-05$ \\
\hline $\mathrm{Ni}+2$ & Nickel & $1.46 \mathrm{E}-03$ & $5.55 \mathrm{E}-03$ & 4.35E-03 & $3.80 \mathrm{E}-03$ \\
\hline $\mathrm{Nb}+5$ & Niobium & $3.18 \mathrm{E}-08$ & $1.86 \mathrm{E}-06$ & 2.19E-06 & $1.37 \mathrm{E}-06$ \\
\hline NO3-1 & Nitrate & $5.05 \mathrm{E}+00$ & $6.34 \mathrm{E}+00$ & $7.44 \mathrm{E}+00$ & $6.29 \mathrm{E}+00$ \\
\hline $\mathrm{Pd}+4$ & Palladium & $1.77 \mathrm{E}-06$ & 4.09E-06 & 4.26E-06 & $3.39 \mathrm{E}-06$ \\
\hline PO4-3 & Phosphate & & $5.82 \mathrm{E}-03$ & $1.37 \mathrm{E}-02$ & $6.58 \mathrm{E}-03$ \\
\hline $\mathrm{Pu}+4$ & Plutonium & $5.47 \mathrm{E}-06$ & $6.36 \mathrm{E}-06$ & $6.82 \mathrm{E}-06$ & $6.22 \mathrm{E}-06$ \\
\hline $\mathrm{Po}+4$ & Polonium & $1.08 \mathrm{E}-18$ & $2.18 \mathrm{E}-18$ & $2.71 \mathrm{E}-18$ & 2.00E-18 \\
\hline $\mathrm{K}+1$ & Potassium & $1.81 \mathrm{E}-01$ & 2.29E-01 & $1.59 \mathrm{E}-01$ & $1.90 \mathrm{E}-01$ \\
\hline $\mathrm{Pr}+4$ & Praseodymium & $4.17 \mathrm{E}-06$ & $9.66 \mathrm{E}-06$ & $1.02 \mathrm{E}-05$ & 8.03E-06 \\
\hline $\mathrm{Pm}+3$ & Promethium & $1.53 \mathrm{E}-09$ & $3.08 \mathrm{E}-09$ & 3.84E-09 & 2.83E-09 \\
\hline $\mathrm{Pa}+4$ & Protactinium & 4.25E-12 & $8.59 \mathrm{E}-12$ & $1.07 \mathrm{E}-11$ & 7.89E-12 \\
\hline $\mathrm{Ra}+2$ & Radium & $1.52 \mathrm{E}-14$ & 3.07E-14 & 3.82E-14 & 2.81E-14 \\
\hline $\mathrm{Rn}$ & Radon & $1.14 \mathrm{E}-19$ & $2.30 \mathrm{E}-19$ & $2.87 \mathrm{E}-19$ & $2.11 \mathrm{E}-19$ \\
\hline $\mathrm{Rh}+4$ & Rhodium & $1.83 \mathrm{E}-06$ & 4.23E-06 & 4.45E-06 & $3.52 \mathrm{E}-06$ \\
\hline $\mathrm{Rb}+1$ & Rubidium & $2.80 \mathrm{E}-06$ & $6.47 \mathrm{E}-06$ & $6.81 \mathrm{E}-06$ & $5.39 \mathrm{E}-06$ \\
\hline $\mathrm{Ru}+3$ & Ruthenium & $8.54 \mathrm{E}-06$ & $2.63 \mathrm{E}-05$ & 2.32E-05 & $1.94 \mathrm{E}-05$ \\
\hline $\mathrm{Sm}+3$ & Samarium & $2.87 \mathrm{E}-06$ & $6.64 \mathrm{E}-06$ & $6.98 \mathrm{E}-06$ & $5.52 \mathrm{E}-06$ \\
\hline $\mathrm{Se}+2$ & Selenium & 1.02E-05 & $1.27 \mathrm{E}-04$ & $8.84 \mathrm{E}-06$ & 4.85E-05 \\
\hline $\mathrm{Si}+4$ & Silicon & & $1.58 \mathrm{E}-03$ & $1.53 \mathrm{E}-04$ & $5.77 \mathrm{E}-04$ \\
\hline $\mathrm{Ag}+1$ & Silver & 4.37E-06 & $1.17 \mathrm{E}-04$ & $2.51 \mathrm{E}-05$ & $4.88 \mathrm{E}-05$ \\
\hline $\mathrm{Na}+1$ & Sodium & $1.97 \mathrm{E}+00$ & $1.59 \mathrm{E}+00$ & $1.70 \mathrm{E}+00$ & $1.75 \mathrm{E}+00$ \\
\hline $\mathrm{Sr}+2$ & Strontium & 4.95E-06 & $1.14 \mathrm{E}-05$ & $1.20 \mathrm{E}-05$ & $9.48 \mathrm{E}-06$ \\
\hline SO4-2 & Sulfate & 4.22E-02 & $5.88 \mathrm{E}-02$ & $4.58 \mathrm{E}-02$ & 4.90E-02 \\
\hline $\mathrm{Tc}+7$ & Technetium & 2.80E-06 & $6.80 \mathrm{E}-06$ & 7.30E-06 & 5.66E-06 \\
\hline $\mathrm{Te}+6$ & Tellurium & $1.44 \mathrm{E}-06$ & $3.34 \mathrm{E}-06$ & $3.52 \mathrm{E}-06$ & $2.78 \mathrm{E}-06$ \\
\hline $\mathrm{Tb}+4$ & Terbium & 1.06E-09 & $2.45 \mathrm{E}-09$ & $2.58 \mathrm{E}-09$ & 2.04E-09 \\
\hline $\mathrm{Tl}+3$ & Thallium & $2.89 \mathrm{E}-20$ & 4.15E-07 & $1.43 \mathrm{E}-06$ & $6.24 \mathrm{E}-07$ \\
\hline $\mathrm{Th}+4$ & Thorium & $9.34 \mathrm{E}-11$ & $1.89 \mathrm{E}-10$ & $2.35 \mathrm{E}-10$ & $1.73 \mathrm{E}-10$ \\
\hline $\mathrm{Tm}+3$ & Thulium & $2.55 \mathrm{E}-15$ & $5.91 \mathrm{E}-15$ & $6.22 \mathrm{E}-15$ & 4.91E-15 \\
\hline $\mathrm{Sn}+4$ & Tin & $1.78 \mathrm{E}-07$ & 4.39E-07 & 9.94E-07 & $5.43 \mathrm{E}-07$ \\
\hline $\mathrm{Ti}+4$ & Titanium & & & $2.34 \mathrm{E}-06$ & 7.97E-07 \\
\hline $\mathrm{U}+4$ & Uranium & $1.48 \mathrm{E}-04$ & 3.31E-04 & 4.04E-04 & $2.96 \mathrm{E}-04$ \\
\hline $\mathrm{V}+5$ & Vanadium & & $2.12 \mathrm{E}-06$ & $8.38 \mathrm{E}-06$ & $3.56 \mathrm{E}-06$ \\
\hline $\mathrm{Yb}+3$ & Ytterbium & $4.30 \mathrm{E}-16$ & 9.96E-16 & $1.05 \mathrm{E}-15$ & $8.29 \mathrm{E}-16$ \\
\hline $\mathrm{Y}+3$ & Yttrium & 3.44E-06 & 7.96E-06 & $8.38 \mathrm{E}-06$ & $6.62 \mathrm{E}-06$ \\
\hline $\mathrm{Zn}+2$ & Zinc & & $4.96 \mathrm{E}-05$ & $1.19 \mathrm{E}-04$ & $5.71 \mathrm{E}-05$ \\
\hline $\mathrm{Zr}+2$ & Zirconium & $1.10 \mathrm{E}-03$ & $1.87 \mathrm{E}-02$ & $2.03 \mathrm{E}-02$ & $1.35 \mathrm{E}-02$ \\
\hline
\end{tabular}




\section{TABLE V: REPRESENTATIVE GLASS COMPOSITION}

$\underline{\text { Waste Species }}$

$\begin{array}{ll}\text { Aluminum } & \mathrm{Al} \\ \text { Arsenic } & \mathrm{As} \\ \text { Barium } & \mathrm{Ba} \\ \text { Beryllium } & \mathrm{Be} \\ \text { Boron } & \mathrm{B} \\ \text { Cadmium } & \mathrm{Cd} \\ \text { Calcium } & \mathrm{Ca} \\ \text { Cerium } & \mathrm{Ce} \\ \text { Chromium } & \mathrm{Cr} \\ \text { Cobalt } & \mathrm{Co} \\ \text { Copper } & \mathrm{Cu} \\ \text { Gadolinium } & \mathrm{Gd} \\ \text { Iron } & \mathrm{Fe} \\ \text { Lead } & \mathrm{Pb} \\ \text { Lithium } & \mathrm{Li} \\ \text { Magnesium } & \mathrm{Mg} \\ \text { Manganese } & \mathrm{Mn} \\ \text { Mercury } & \mathrm{Hg} \\ \text { Molybdenum } & \mathrm{Mo} \\ \text { Nickel } & \mathrm{Ni} \\ \text { Potassium } & \mathrm{K} \\ \text { Ruthenium } & \mathrm{Ru} \\ \text { Sodium } & \mathrm{Na} \\ \text { Strontium } & \mathrm{Sr} \\ \text { Titanium } & \mathrm{Ti} \\ \text { Uranium } & \mathrm{U} \\ \text { Zinc } & \mathrm{Zn} \\ \text { Zirconium } & \mathrm{Zr} \\ \text { Chloride } & \mathrm{Cl} \\ \text { Fluoride } & \mathrm{F} \\ \text { lodide } & \mathrm{I} \\ \text { Nitrate } & \mathrm{NO} 3 \\ \text { Phosphate } & \mathrm{P} 2 \mathrm{O} 5 \\ \text { Sulfate } & \mathrm{SO} 3 \\ & \\ \text { Radionuclides } & \\ \text { Cesium } & \mathrm{Cs} \\ \text { Europium } & \mathrm{Eu} \\ \text { Americium(III) } & \mathrm{Am} \\ \text { Tecnecium-99 } & \mathrm{Tc} \\ \text { Plutonium (IV) } & \mathrm{Pu} \\ & \end{array}$

Frit

$\begin{array}{lllc}\text { Boron } & & \text { Oxide form } & \text { (as \% of Frit) } \\ \text { Calcium } & \mathrm{B} & \mathrm{B} 2 \mathrm{O} 3 & 15 \% \\ \text { Iron } & \mathrm{Ca} & \mathrm{CaO} & 5 \% \\ \text { Lithium } & \mathrm{Fe} & \mathrm{Fe} 2 \mathrm{O} 3 & 10 \% \\ \text { Silicon } & \mathrm{Li} & \mathrm{Li} 2 \mathrm{O} & 5 \% \\ & \mathrm{Si} & \mathrm{SiO} 2 & 65 \%\end{array}$

Weight
Percentage $\%$ of Frit)

\begin{tabular}{|c|c|}
\hline $\begin{array}{c}\text { Oxide form } \\
\text { (Anion form in Glass) }\end{array}$ & Mass Fraction Oxides in Glass \\
\hline $\mathrm{Al} 2 \mathrm{O} 3$ & 0.083890392 \\
\hline As2O3 & $6.10428 E-05$ \\
\hline $\mathrm{BaO}$ & 2.11297E-05 \\
\hline $\mathrm{BeO}$ & 4.8017E-07 \\
\hline $\mathrm{B} 2 \mathrm{O} 3$ & 0.001058636 \\
\hline $\mathrm{CdO}$ & 0.00023939 \\
\hline $\mathrm{CaO}$ & 0.006652719 \\
\hline $\mathrm{CeO} 2$ & $1.00551 \mathrm{E}-05$ \\
\hline $\mathrm{Cr} 2 \mathrm{O} 3$ & 0.000629842 \\
\hline Co3O4 & 5.73356E-06 \\
\hline $\mathrm{CuO}$ & 0.000137062 \\
\hline $\mathrm{Gd} 2 \mathrm{O} 3$ & 7.94293E-05 \\
\hline $\mathrm{Fe} 2 \mathrm{O} 3$ & 0.004287623 \\
\hline $\mathrm{PbO}$ & 0.000720884 \\
\hline Li2O & $1.25314 \mathrm{E}-05$ \\
\hline $\mathrm{MgO}$ & 0.001199333 \\
\hline $\mathrm{MnO} 2$ & 0.003031035 \\
\hline $\mathrm{HgO}$ & 0 \\
\hline $\mathrm{MoO} 3$ & $6.85832 \mathrm{E}-05$ \\
\hline $\mathrm{NiO}$ & 0.000271763 \\
\hline $\mathrm{K} 2 \mathrm{O}$ & 0.022844955 \\
\hline $\mathrm{RuO} 2$ & $2.04957 \mathrm{E}-05$ \\
\hline $\mathrm{Na} 2 \mathrm{O}$ & 0.157642155 \\
\hline $\mathrm{SrO}$ & 3.05082E-05 \\
\hline $\mathrm{TiO} 2$ & 1.14118E-05 \\
\hline U308 & 0.000699675 \\
\hline $\mathrm{ZnO}$ & 0.000211106 \\
\hline $\mathrm{ZrO} 2$ & 1.92664E-05 \\
\hline $\mathrm{Cl}$ & 0.002584381 \\
\hline$F$ & 0.000403192 \\
\hline I & 4.2051E-05 \\
\hline NO3 & 0 \\
\hline P2O5 & 0.002403788 \\
\hline SO3 & 0.010698003 \\
\hline $\mathrm{Cs} 2 \mathrm{O}$ & 2.85457E-06 \\
\hline Eu2O3 & $1.43948 \mathrm{E}-08$ \\
\hline $\mathrm{Am} 2 \mathrm{O} 3$ & $7.53245 \mathrm{E}-08$ \\
\hline $\mathrm{TcO} 2$ & 2.0004E-06 \\
\hline $\mathrm{PuO} 2$ & $\underline{6.40331 \mathrm{E}-06}$ \\
\hline Sum of Waste in Glass & 0.3 \\
\hline
\end{tabular}

Mass Fraction Oxides in Glass

0.105

0.035

0.07

0.035

$\underline{0.455}$

Sum of Frit in Glass 


\section{TABLE VI: MISCELLANEOUS MASS BALANCE ASSUMPTIONS}

\begin{tabular}{|c|c|}
\hline Evaporator & - Batch process terminates when solution reaches a specific gravity of 1.33 \\
\hline Acid Fractionator & - Bottoms stream is $10 \mathrm{M}$ nitric acid \\
\hline Melter & $\begin{array}{l}\text { - Steady-state glass production rate of } 127 \mathrm{~kg} / \mathrm{hr} \\
\text { - Glass waste loading - } 30 \% \text { wt. of the glass is composed of nonvolatile } \\
\text { species in the waste on an oxide basis (a representative glass composition } \\
\text { is shown in Table } \mathrm{V} \text { ) } \\
\text { - A limit of } 33 \% \mathrm{Fe}+3 \text { to } \mathrm{Fe}+2 \text { reduction determines the amount of sugar } \\
\text { [C6H12O6] added (this corresponds to about } 141 \mathrm{~g} \text { sugar per liter of } \\
\text { waste) } \\
\text { - Frit will comprise } 70 \% \text { wt. of the glass, and its composition is as shown in } \\
\text { Table } \mathrm{V} \text {. } \\
\text { - } 45 \mathrm{SCM} / \mathrm{Hr} \text { of input air }\end{array}$ \\
\hline Ejector Fume Scrubber & - $145 \mathrm{SCM} / \mathrm{Hr}$ of air (pressure control) added to input gas stream \\
\hline Caustic Scrubber & $\begin{array}{l}\text { - } \\
\text { - } \\
\text { Mauntain pH of } 7.0\end{array}$ \\
\hline Noxidizer & $\begin{array}{ll}\text { - } & \text { Fuel is kerosene } \\
\text { - } & \text { NOx conversion in denoxidizer complies with MACT } \\
\text { - } & \text { O2 in effluent is } 2 \% \text { on a dry basis } \\
\text { - } & \text { heat loss to the environment is } \sim 10 \%\end{array}$ \\
\hline SCR & - Ammonia feed is pure NH3 gas \\
\hline Settling Tank & - $2 \%$ of liquid passes to ion exchange \\
\hline Scrub Purge Grout Mix Tank & $\begin{array}{l}\text { - Grout waste loading - } 42.5 \% \mathrm{wt} \\
\text { - } 50 \% \text { wt. } \mathrm{NaOH} \text { solution added to maintain } \mathrm{pH} 12.0 \\
\text { Representative recipe is as follows: } 101 \mathrm{~kg} \mathrm{NaOH} \text { solution, } 117 \mathrm{~kg} \text { solid } \\
\mathrm{Ca}(\mathrm{OH}) 2,693 \mathrm{~kg} \text { liquid waste, } 72 \mathrm{~kg} \text { portland cement, and } 647 \mathrm{~kg} \text { blast } \\
\text { furnace slag }\end{array}$ \\
\hline
\end{tabular}


FIGURES

Figure I: Process Configurations 


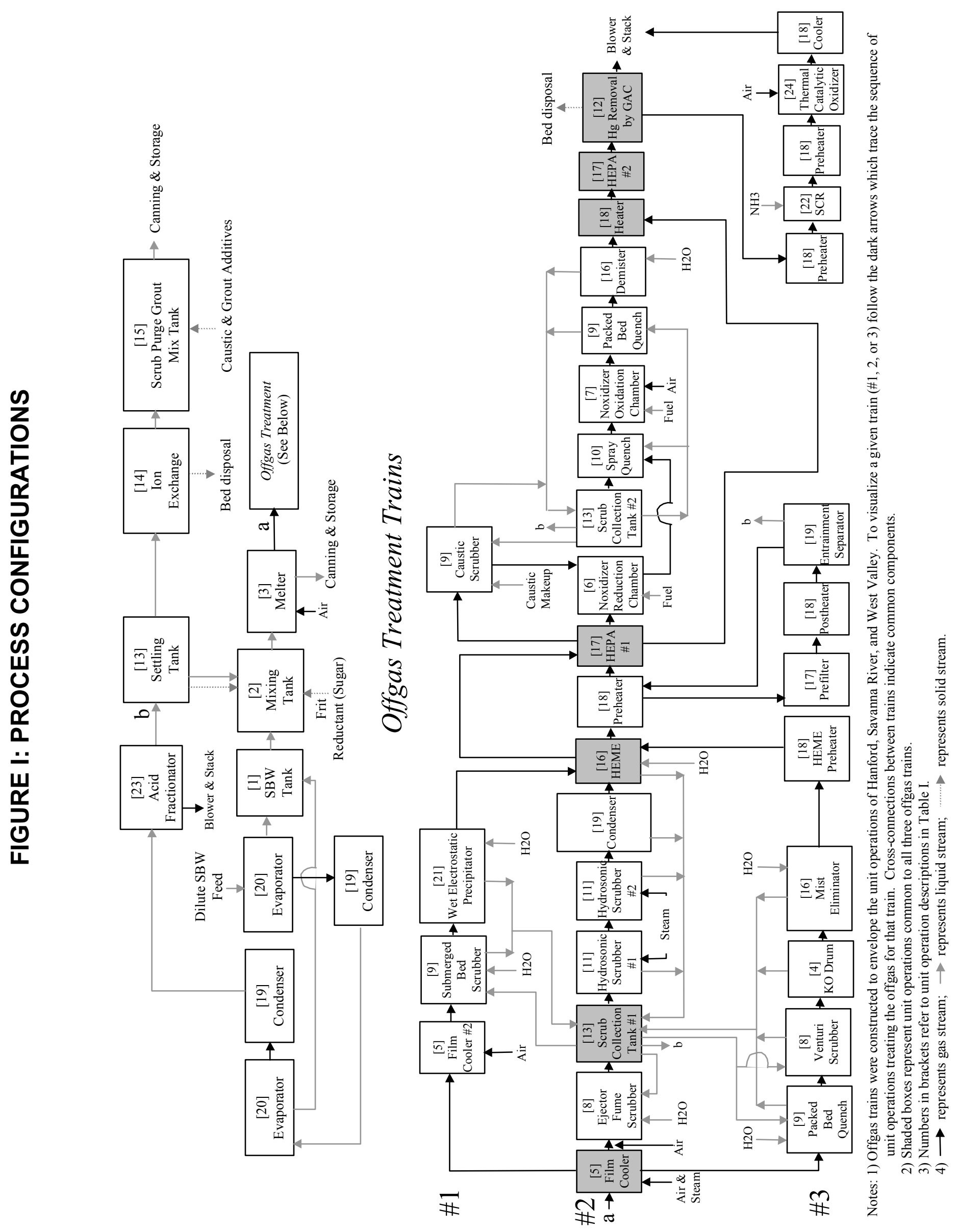




\section{REFERENCES}

Barnes, C.M. (2000a), File named "Dir Vit Unit Operations.doc, "An attachment to Lotus Notes memo to D.D. Taylor of INEEL, 10/19.

Barnes, C.M. (2000b), Feed Composition for Sodium-Bearing Waste Treatment Process, INEEL/EXT2000-01378, Revision 0, October, pp. 12-16.

Barnes, Charles, Lance Lauerhass, Todd Nichols, and Dean Taylor (2000), INEEL Letter to A.L. Olson and C.A. Musick, Simulation of HLW Treatment Process Trains at NEEL - Scope -, CMB-1000, November 13.

Langowski, M.H., H.G. Darab, and P.A. Smith (1996), Volatility Literature of Chlorine, Iodine, Cesium, Strontium, Technetium, and Rhenium; Technetium and Rhenium Volatility Testing, PNNL11052/UC-512, March.

Nichols, Todd (2000), INEEL E-mail to A.L. Olson, C.M. Barnes, L. Lauerhass, D.D. Taylor, L.L. Simmons, J.D. Christian, and N. Hutson (Savanna River Site), MINUTES OF TTP PLANNING MEETING BETWEEN SRS \& INEEL -10/25/00, November 1. Copy accessible from Document Control Center for INEEL, Applied Technology, Organization 3330.

Patterson, M. (1999), Light Duty Utility Arm Deployment in Tank WM-188, INEEL/EXT-99-01302, December.

Savannah River Technology Center (2001), Software QA Plan for INEEL Vitrification Flowsheet Modeling, WSRC-RP-2001-00207, Revision 0, January 31.

Schindler, R. E. (1998), Evaporation of Blends of HLLWE Condensate with CPP-603 Basin Water, INEEL Letter to F.S. Ward, SCHI-26-98, November 18, p.9.

Soelberg, Nick (2001), Personal communication to D.D. Taylor of INEEL, January 18.

Welland, H. (1997), NWCF Process Modification for Sodium-Bearing Waste Project Conceptual Design, INEL/INT-97-00075. 